\title{
Kannan-type contractions and fixed points in uniform spaces
}

\author{
Kazimierz Włodarczyk ${ }^{*}$ and Robert Plebaniak
}

\author{
* Correspondence: wlkzxa@math. \\ uni.lodz.pl \\ Department of Nonlinear Analysis, \\ Faculty of Mathematics and \\ Computer Science, University of \\ Łódź, Banacha 22, 90-238 Łódź, \\ Poland
}

\begin{abstract}
In uniform spaces, using $\mathcal{J}$-families of generalized pseudodistances, we construct four kinds of contractions of Kannan type and, by techniques based on these generalized pseudodistances, we prove fixed point theorems for such contractions. The results are new in uniform and locally convex spaces and even in metric spaces. Examples are given.

MSC: 47H10; 47H09; 54E15; 46A03; 54E35.
\end{abstract}

Keywords: uniform space, metric space, J-family of generalized pseudodistances; contractions of Kannan type, fixed point; iterative approximation

\section{Introduction}

Given a space $X, \operatorname{Fix}(T)$ denotes the set of fixed points of $T: X \rightarrow X$, i.e. $\operatorname{Fix}(T)=\{w \in$ $X: w=T(w)\}$. For $v^{0}$ any point of $X$, by $\left(v^{m}: m \in\{0\} \cup \mathbb{N}\right)$ we mean the sequence of iteration of $T: X \rightarrow X$ starting at $v^{0}$, i.e. $\forall_{m \in\{0\} \cup \mathbb{N}}\left\{v^{m}=T^{[m]}\left(v^{0}\right)\right\}$.

Recall that maps satisfying the conditions (B) and (K) that are presented in Theorems 1.1 and 1.2 below are called in literature Banach contractions and Kannan contractions, respectively, and first arose in works $[1,2]$ and $[3,4]$, respectively.

Theorem 1.1 [1,2]Let $(X, d)$ be a complete metric space. If $T: X \rightarrow X$ satisfies

$$
\text { (B) } \exists_{\lambda \in[0,1)} \forall_{x, y \in X}\{d(T(x), T(y)) \leq \lambda d(x, y)\} \text { ， }
$$

then: (a) Thas a unique fixed point $w$ in $X$; and (b) $\forall_{u^{0} \in X}\left\{\lim _{m \rightarrow \infty} u^{m}=w\right\}$.

Theorem 1.2 [3] Let $(X, d)$ be a complete metric space. If $T: X \rightarrow X$ satisfies $(K) \exists_{\eta \in}$ $[0,1 / 2) \forall_{x, y \in X}\{d(T(x), T(y)) \leq \eta[d(T(x), x)+d(T(y), y)]\}$,

then: (a) Thas a unique fixed point $w$ in $X$; and (b) $\forall_{u^{0} \in X}\left\{\lim _{m \rightarrow \infty} u^{m}=w\right\}$.

Theorem 1.3 [4] Let $(X, d)$ be a metric space. Assume that: (i) $T: X \rightarrow X$ satisfies (K); (ii) there exists $w \in X$ such that $T$ is continuous at a point $w$; and (iii) there exists a point $v^{0} \in X$ such that the sequence $\left(v^{m}: m \in\{0\} \cup \mathbb{N}\right)$ has a subsequence $\left(v^{m k}: k \in\right.$ $\{0\} \cup \mathbb{N}$ ) satisfying $\lim _{k \rightarrow \infty} v^{m_{k}}=w$. Then, $w$ is a unique fixed point of $T$ in $X$.

A great number of applications and extensions of these results have appeared in the literature and plays an important role in nonlinear analysis. The different line of research focuses on the study of the following interesting aspects of fixed point theory in metric spaces and has intensified in the past few decades: (a) the existence and uniqueness of fixed points of various generalizations of Banach and Kannan contractions; (b) the similarity between Banach and Kannan contractions; and (c) the interplay 
between metric completeness and the existence of fixed points of Banach and Kannan contractions. These aspects have been successfully studied in various papers; see, for example, [5-21] and references therein.

It is interesting that Theorem 1.2 is independent of Theorem 1.1 that every Banach contraction and every Kannan contraction on a complete metric space has a unique fixed point and that in Theorem 1.3 the completeness of the metric space is omitted. Clearly, Banach contractions are always continuous but Kannan contractions are not necessarily continuous. Next, it is worth noticing that Theorem 1.2 is not an extension of Theorem 1.1. In [5], it is constructed an example of noncomplete metric space $X$ such that each Banach contraction $T: X \rightarrow X$ has a fixed point which implies that Theorem 1.1 does not characterize metric completeness. In [6], it is proved that a metric space $X$ is complete if and only if every Kannan contraction $T: X \rightarrow X$ has a fixed point which implies that Theorem 1.2 characterizes the metric completeness. Similarity between Banach and Kannan contractions may be seen in $[7,8]$. In complete metric spaces $(X, d), w$-distances [9] and $\tau$-distances [10] have found substantial applications in fixed point theory and among others generalizations of Banach and Kannan contractions are introduced, many interesting extensions of Theorems 1.1 and 1.2 to $w$-distances and $\tau$-distances are obtained, and techniques based on these distances are presented (see, for example, [7-17]); $\tau$-distances generalize $w$-distances and metrics $d$.

The above are some of the reasons why in metric spaces the study of Kannan contractions and generalizations of Kannan contractions plays a particularly important part in fixed point theory.

In this article, in uniform spaces, using $\mathcal{J}$-families of generalized pseudodistances, we construct four kinds of contractions of Kannan type (see conditions (C1)-(C4)) and, by techniques based on these generalized pseudodistances, we prove fixed point theorems for such contractions (see Theorems 2.1-2.8). The definitions and the results are new in uniform and locally convex spaces and even in metric spaces. Examples (see Section 12) and some conclusions (see Section 13) are given.

\section{Statement of main results}

Let $X$ be a Hausdorff uniform space with uniformity defined by a saturated family $\mathcal{D}=\left\{d_{\alpha}: X^{2} \rightarrow[0, \infty), \alpha \in \mathcal{A}\right\}$ of pseudometrics $d_{\alpha}, \alpha \in \mathcal{A}$, uniformly continuous on $X^{2}$. The notion of $\mathcal{J}$-family of generalized pseudodistances on $X$ is as follows:

Definition 2.1 Let $X$ be a uniform space. The family $\mathcal{J}=\left\{J_{\alpha}, \alpha \in \mathcal{A}\right\}$ of maps $J_{\alpha}$ : $X^{2} \rightarrow[0, \infty), \alpha \in \mathcal{A}$, is said to be a $\mathcal{J}$-family of generalized pseudodistances on $X$ ( $\mathcal{J}$-family, for short) if the following two conditions hold: $(\mathcal{J} 1) \forall_{\alpha \in \mathcal{A}} \forall_{x, y, z \in X}\left\{J_{\alpha}(x, z) \leq J_{\alpha}(x, y)+J_{\alpha}(y, z)\right\}$; and

$(\mathcal{J} 2)$ For any sequences $\left(x_{m}: m \in \mathbb{N}\right)$ and $\left(y_{m}: m \in \mathbb{N}\right)$ in $X$ such that

$$
\forall_{\alpha \in \mathcal{A}}\left\{\lim _{n \rightarrow \infty} \sup _{m>n} J_{\alpha}\left(x_{n}, x_{m}\right)=0\right\}
$$

and

$$
\forall_{\alpha \in \mathcal{A}}\left\{\lim _{m \rightarrow \infty} J_{\alpha}\left(x_{m}, y_{m}\right)=0\right\},
$$


the following holds:

$$
\forall \forall_{\alpha \in \mathcal{A}}\left\{\lim _{m \rightarrow \infty} d_{\alpha}\left(x_{m}, y_{m}\right)=0\right\} .
$$

Remark 2.1 Let $X$ be a uniform space.

(a) Let $\mathcal{J}=\left\{J_{\alpha}: X^{2} \rightarrow[0, \infty), \alpha \in \mathcal{A}\right)$ be a $\mathcal{J}$-family. if $\forall_{\alpha \in \mathcal{A}} \forall_{x \in x}\left\{J_{\alpha}(x, x)=0\right\}$, then, for each $\alpha \in \mathcal{A}, J_{\alpha}$ is quasi-pseudometric. Examples of $\mathcal{J}$-families such that the maps $J_{\alpha}, \alpha \in \mathcal{A}$ are not quasi-pseudometrics are given in Section 12.

(b) The family $\mathcal{J}=\mathcal{D}$ is a $\mathcal{J}$-family on $X$.

It is the purpose of the present paper to prove the following results.

Theorem 2.1 Let $X$ be a Hausdorff uniform space and assume that the map $T: X \rightarrow$ $X$ and the $\mathcal{J}$-family $\mathcal{J}=\left\{J_{\alpha}: X^{2} \rightarrow[0, \infty), \alpha \in \mathcal{A}\right\}$ on $\quad X \quad$ satisfy $\quad(C 1)$ $\forall_{\alpha \in \mathcal{A}} \exists_{\eta_{\alpha} \in[0,1 / 2)} \forall_{x, y \in X}\left\{J_{\alpha}(T(x), T(y)) \leq \eta_{\alpha}\left[J_{\alpha}(T(x), x)+J_{\alpha}(T(y), y)\right]\right\}$ and, additionally,

(D1) $\exists_{v^{0}, \omega \in X} \forall_{\alpha \in \mathcal{A}}\left\{\lim _{m \rightarrow \infty} J_{\alpha}\left(v^{m}, w\right)=0\right\}$.

Then: (a) $T$ has a unique fixed point $w$ in $X$; (b) $\forall_{u^{0} \in X}\left\{\lim _{m \rightarrow \infty} u^{m}=w\right\}$; and (c) $\forall \forall_{\alpha \in \mathcal{A}}\left\{J_{\alpha}(w, w)=0\right\}$.

Theorem 2.2 Let $X$ be a Hausdorff uniform space and assume that the map $T: X \rightarrow$ $X$ and the $\mathcal{J}$-family $\mathcal{J}=\left\{J_{\alpha}: X^{2} \rightarrow[0, \infty), \alpha \in \mathcal{A}\right\}$ on $X$ satisfy at least one of the following three conditions:

(C2) $\forall_{\alpha \in \mathcal{A}} \exists_{\eta_{\alpha} \in[0,1 / 2)} \forall_{x, y \in X}\left\{J_{\alpha}(T(x), T(y)) \leq \eta_{\alpha}\left[J_{\alpha}(T(x), x)+J_{\alpha}(T(y)), y\right]\right\}$,

(C3) $\forall_{\alpha \in \mathcal{A}} \exists_{\eta_{\alpha} \in[0,1 / 2)} \forall_{x, y \in X}\left\{J_{\alpha}(T(x), T(y)) \leq \eta_{\alpha}\left[J_{\alpha}(x, T(x))+J_{\alpha}(y, T(y))\right]\right\}$,

(c4) $\forall_{\alpha \in \mathcal{A}} \exists_{\eta_{\alpha} \in[0,1 / 2)} \forall_{x, y \in X}\left\{J_{\alpha}(T(x), T(y)) \leq \eta_{\alpha}\left[J_{\alpha}(x, T(x))+J_{\alpha}(T(y), y)\right]\right\}$,

and, additionally,

(D2) $\exists_{v^{0}, \omega \in X} \forall_{\alpha \in \mathcal{A}}\left\{\lim _{m \rightarrow \infty} J_{\alpha}\left(v^{m}, w\right)=\lim _{m \rightarrow \infty} J_{\alpha}\left(w, v^{m}\right)=0\right\}$.

Then: (a) T has a unique fixed point $w$ in $X$; (b) $\forall_{u^{0} \in X}\left\{\lim _{m \rightarrow \infty} u^{m}=w\right\}$; and (c) $\forall_{\alpha \in \mathcal{A}}\left\{J_{\alpha}(w, w)=0\right\}$.

It is worth noticing that conditions (C1)-(C4) are different and conditions (D1) and (D2) are different since the $\mathcal{J}$-family is not symmetric.

Clearly, (D1) include (D2). The following theorem shows that with some additional conditions the converse holds.

Theorem 2.3 Let $X$ be a Hausdorff uniform space and assume that the map $T: X \rightarrow$ $X$ and the $\mathcal{J}$-family $\mathcal{J}=\left\{J_{\alpha}: X^{2} \rightarrow[0, \infty), \alpha \in \mathcal{A}\right\}$ on $X$ satisfy at least one of the conditions (C2)-(C4) and, additionally, condition (D1) and at least one of the following conditions (D3)-(D6):

(D3) $\forall_{v^{0}, w \in X}\left\{\lim _{m \rightarrow \infty} v^{m}=w \Rightarrow \exists_{q \in \mathbb{N}}\left\{T^{[q]}(w)=w\right\}\right\}$,

(D4) $\forall_{v^{0}, w \in X}\left\{\lim _{m \rightarrow \infty} v^{m}=w \Rightarrow \exists_{q \in \mathbb{N}}\left\{T^{[q]}\right.\right.$ is continuous at a point $\left.\left.w\right\}\right\}$,

(D5) $\forall_{v^{0}, w \in X}\left\{\lim _{m \rightarrow \infty} v^{m}=w \Rightarrow \exists_{q \in \mathbb{N}}\left\{\lim _{m \rightarrow \infty} T^{[q]}\left(v^{m}\right)=T^{[q]}(w)\right\}\right\}$,

(D6) $\forall_{v^{0}, w \in X}\left\{\lim _{m \rightarrow \infty} v^{m}=w \Rightarrow \exists_{q \in \mathbb{N}} \forall_{\alpha \in \mathcal{A}}\left\{\lim _{m \rightarrow \infty} J_{\alpha}\left(T^{[q]}\left(v^{m}\right), T^{[q]}(w)\right)=0\right\}\right\}$.

Then: (a) Thas a unique fixed point $w$ in $X$; (b) $\forall_{u^{0} \in X}\left\{\lim _{m \rightarrow \infty} u^{m}=w\right\}$; and (c) $\forall_{\alpha \in A}\left\{J_{\alpha}(w, w)=0\right\}$.

The following theorem shows that if we assume that the uniform space is sequentially complete, then the conditions (D1) and (D2) can be omitted.

Theorem 2.4 Let $X$ be a Hausdorff sequentially complete uniform space and assume that the map $T: X \rightarrow X$ and the $\mathcal{J}$-family $\mathcal{J}=\left\{J_{\alpha}: X^{2} \rightarrow[0, \infty), \alpha \in \mathcal{A}\right\}$ on $X$ satisfy 
at least one of the conditions (C1)-(C4) and, additionally, at least one of the conditions (D3)-(D6). Then: (a) $T$ has a unique fixed point $w$ in $X$; (b) $\forall_{u^{0} \in X}\left\{\lim _{m \rightarrow \infty} u^{m}=w\right\}$; and (c) $\forall_{\alpha \in \mathcal{A}}\left\{J_{\alpha}(w, w)=0\right\}$.

We now introduce the concept of $\mathcal{J}$-admissible maps and in the following result these maps will be used to extend Theorem 2.4 to the uniform spaces which are not sequentially complete and without any conditions (D1)-(D6).

Definition 2.2 Let $X$ be a Hausdorff uniform space and let $\mathcal{J}=\left\{J_{\alpha}: X^{2} \rightarrow[0, \infty), \alpha \in \mathcal{A}\right)$ be a $\mathcal{J}$-family on $X$. We say that $T: X \rightarrow X$ is $\mathcal{J}$-admissible if for each $u^{0} \in X$ satisfying $\forall_{\alpha \in \mathcal{A}}\left\{\lim _{n \rightarrow \infty} \sup _{m>n} J_{\alpha}\left(u^{n}, u^{m}\right)=0\right\}$ there exists $w \in X$ such that $\forall_{\alpha \in \mathcal{A}}\left\{\lim _{m \rightarrow \infty} J_{\alpha}\left(u^{m}, w\right)=\lim _{m \rightarrow \infty} J_{\alpha}\left(w, u^{m}\right)=0\right\}$.

Theorem 2.5 Let $X$ be a Hausdorff uniform space and $\mathcal{J}=\left\{J_{\alpha}: X^{2} \rightarrow[0, \infty), \alpha \in \mathcal{A}\right\}$ be the $\mathcal{J}$-family on $X$. Let the map $T: X \rightarrow X$ be $\mathcal{J}$-admissible and assume that $T$ and $\mathcal{J}$ satisfy at least one of the conditions (C1)-(C4). Then: (a) $T$ has a unique fixed point $w$ in $X$; (b) $\forall_{u^{0} \in X}\left\{\lim _{m \rightarrow \infty} u^{m}=w\right\}$; and (c) $\forall_{\alpha \in \mathcal{A}}\left\{J_{\alpha}(w, w)=0\right\}$.

Also, the following uniqueness results hold.

Theorem 2.6 Let $X$ be a Hausdorff uniform space and assume that the map $T: X \rightarrow$ $X$ and the $\mathcal{J}$-family $\mathcal{J}=\left\{J_{\alpha}: X^{2} \rightarrow[0, \infty), \alpha \in \mathcal{A}\right\}$ on $X$ satisfy at least one of the conditions (C1)-(C4) and, additionally, the following conditions (D7) and (D8):

(D7) There exist $q \in \mathbb{N}$ and $w \in X$ such that $T^{[q]}$ is continuous at a point $w$;

(D8) There exists a point $v^{0} \in X$ such that the sequence $\left(v^{m}: m \in\{0\} \cup \mathbb{N}\right)$ has a subsequence $\left(v^{m k}: k \in\{0\} \cup \mathbb{N}\right)$ satisfyinglim $\lim _{k \rightarrow \infty} v^{m_{k}}=w$.

Then: (a) $T$ has a unique fixed point $w$ in $X$; (b) $\lim _{k \rightarrow \infty} v^{m}=w$; and (c) $\forall \forall_{\alpha \in \mathcal{A}}\left\{J_{\alpha}(w, w)=0\right\}$.

Theorem 2.7 Let $X$ be a Hausdorff sequentially complete uniform space and let the map $T: X \rightarrow X$ satisfy the condition

(C5) $\forall_{\alpha \in \mathcal{A}} \exists_{\eta_{\alpha} \in[0,1 / 2)} \forall_{x, y \in X}\left\{d_{\alpha}(T(x), T(\gamma)) \leq \eta_{\alpha}\left[d_{\alpha}(T(x), x)+d_{\alpha}(\gamma, T(y))\right]\right\}$.

Then: (a) $T$ has a unique fixed point $w$ in $X$; and (b) $\forall_{u^{0} \in X}\left\{\lim _{m \rightarrow \infty} u^{m}=w\right\}$.

Theorem 2.8 Let $X$ be a Hausdorff uniform space and assume that the map $T: X \rightarrow$ $X$ satisfies (C5), (D7) and (D8). Then: (a) T has a unique fixed point $w$ in $X$; and (b) $\forall u^{0} \in X\left\{\lim _{m \rightarrow \infty} u^{m}=w\right\}$.

The rest of this article is organized as follows. In Section 3, we prove some auxiliary propositions. In Sections 4-11, we prove Theorems 2.1-2.8, respectively. Section 12 provides examples and comparisons. Section 13 includes some conclusions.

\section{Auxiliary propositions}

In this section, we present some propositions that will be used in Sections 4-11.

Proposition 3.1 Let $X$ be a Hausdorff uniform space and let $\mathcal{J}=\left\{J_{\alpha}: X^{2} \rightarrow[0, \infty), \alpha \in \mathcal{A}\right\} b e$ a $\mathcal{J}$-family. If $x \neq y, x, \quad y \in X$, then $\exists_{\alpha \in \mathcal{A}}\left\{J_{\alpha}(x, y) \neq 0 \vee J_{\alpha}(y, x) \neq 0\right\}$.

Remark 3.1 If $x, y \in X$ and $\forall_{\alpha \in \mathcal{A}}\left\{J_{\alpha}(x, y)=0 \wedge J_{\alpha}(y, x)=0\right\}$, then $x=y$.

Proof of Proposition 3.1. Suppose that $x \neq y$ and $\forall_{\alpha \in \mathcal{A}}\left\{J_{\alpha}(x, y)=0 \wedge J_{\alpha}(y, x)=0\right\}$. Then, $\quad \forall_{\alpha \in \mathcal{A}}\left\{J_{\alpha}(x, x)=0\right\}$, since, by $(\mathcal{J} 1)$, we get $\forall_{\alpha \in \mathcal{A}}\left\{d_{\alpha}(x, y)=0\right\}$. Defining $x_{m}=x$ and $y_{m}=y$ for $m \in \mathbb{N}$, we conclude that (2.1) and 
(2.2) hold. Consequently, by (J2), we get (2.3) which implies $\forall_{\alpha \in \mathcal{A}}\left\{d_{\alpha}(x, y)=0\right\}$. However, $X$ is a Hausdorff and hence, since $x \neq y$, we have. $\exists_{\alpha \in \mathcal{A}}\left\{d_{\alpha}(x, y) \neq 0\right\}$. Contradiction.

Proposition 3.2 Let $X$ be a uniform space and let $\mathcal{J}=\left\{J_{\alpha}: X^{2} \rightarrow[0, \infty), \alpha \in \mathcal{A}\right\}$ be a $\mathcal{J}$-family. Let $\Phi=\left\{\phi_{\alpha}, \alpha \in \mathcal{A}\right\}$ be the family of maps $\varphi_{\alpha}: X \rightarrow[0, \infty), \alpha \in \mathcal{A}$.

(a) The families $\mathcal{W}^{(i)}=\left\{W_{\alpha}^{(i)}: X^{2} \rightarrow[0, \infty), \alpha \in \mathcal{A}\right\}, i=1,2$, where, for each $W_{\alpha}^{(1)}(x, y)=\max \left\{\phi_{\alpha}(x), J_{\alpha}(x, y)\right\}, \quad W_{\alpha}^{(1)}(x, y)=\max \left\{\phi_{\alpha}(x), J_{\alpha}(x, y)\right\}$ and $W_{\alpha}^{(2)}(x, y)=\max \left\{\phi_{\alpha}(y), J_{\alpha}(x, y)\right\}, x, y \in X$, are $\mathcal{J}$-families on $X$.

(b) The families $\mathcal{V}^{(i)}=\left\{V_{\alpha}^{(i)}: X^{2} \rightarrow[0, \infty), \alpha \in \mathcal{A}\right\}, i=1,2$, where for each $V_{\alpha}^{(2)}(x, y)=\phi_{\alpha}(y)+J_{\alpha}(x, y) V_{\alpha}^{(1)}(x, y)=\phi_{\alpha}(x)+J_{\alpha}(x, y)$ and $V_{\alpha}^{(2)}(x, y)=\phi_{\alpha}(y)+J_{\alpha}(x, y)$, $x, y \in X$, are $\mathcal{J}$-families on $X$.

Remark $3.2 \forall_{i \in\{1,2\}} \forall_{\alpha \in \mathcal{A}} \forall_{x, y \in X}\left\{J_{\alpha}(x, y) \leq W_{\alpha}^{(i)}(x, y) \wedge J_{\alpha}(x, y) \leq V_{\alpha}^{(i)}(x, y)\right\}$.

Proof of Proposition 3.2. (a) For each $\alpha \in \mathcal{A}$ and for each $x, y, z \in X$, using (J1) for family $\mathcal{J}, \quad$ we get $\left.W_{\alpha}^{(2)}(x, y)=\max \left\{\phi_{\alpha}(z), J_{\alpha}(x, z)\right\} \leq \max \left\{\phi_{\alpha}(y)+\phi_{\alpha}(z), J_{\alpha}(x, y)+J_{\alpha}(y, z)\right\} \leq W_{\alpha}^{(2)}(x, y)+W_{\alpha}^{(2)}(y, z)\right\}$ and $\left.W_{\alpha}^{(2)}(x, y)=\max \left\{\phi_{\alpha}(z), J_{\alpha}(x, z)\right\} \leq \max \left\{\phi_{\alpha}(y)+\phi_{\alpha}(z), J_{\alpha}(x, y)+J_{\alpha}(y, z)\right\} \leq W_{\alpha}^{(2)}(x, y)+W_{\alpha}^{(2)}(y, z)\right\}$. Therefore, for each $i \in\{1,2\}$, the condition $(\mathcal{J} 1)$ for family $\mathcal{W}^{(i)}$ holds.

Let $i \in\{1,2\}$ be arbitrary and fixed and let $\left(x_{m}: m \in \mathbb{N}\right)$ and $\left(y_{m}: m \in \mathbb{N}\right)$ be arbitrary and fixed sequences in $X$ satisfying $\forall_{\alpha \in \mathcal{A}}\left\{\lim _{n \rightarrow \infty} \sup _{m>n} W_{\alpha}^{(i)}\left(x_{n}, x_{m}\right)=\lim _{m \rightarrow \infty} W_{\alpha}^{(i)}\left(x_{m}, y_{m}\right)=0\right\}$. Then, by Remark 3. 2, we obtain that the. conditions (2.1) and (2.2) for family $\mathcal{J}$ hold and, consequently, since $\mathcal{J}$ is a $\mathcal{J}$-family, by $(\mathcal{J} 2)$, the condition (2.3) is satisfied, i.e. $\forall_{\alpha \in \mathcal{A}}\left\{\lim _{m \rightarrow \infty} d_{\alpha}\left(x_{m}, y_{m}\right)=0\right\}$ which gives that $(\mathcal{J} 2)$ for family $\mathcal{W}^{(i)}$ holds.

Therefore, for each $i \in\{1,2\}, \mathcal{W}^{(i)}$ is $\mathcal{J}$-family.

(b) Using ( $\mathcal{J} 1)$ for family $\mathcal{J}$, we obtain that, for each $\alpha \in \mathcal{A}$ and for each $x, y, z, \in$ $X, \quad V_{\alpha}^{(1)}(x, z)=\phi_{\alpha}(x)+J_{\alpha}(x, z) \leq \phi_{\alpha}(x)+J_{\alpha}(x, y)+\phi_{\alpha}(\gamma)+J_{\alpha}(y, z)=V_{\alpha}^{(1)}(x, y)+V_{\alpha}^{(1)}(y, z)$, and $V_{\alpha}^{(2)}(x, z)=\phi_{\alpha}(z)+J_{\alpha}(x, z) \leq \phi_{\alpha}(y)+J_{\alpha}(x, y)+\phi_{\alpha}(z)+J_{\alpha}(y, z)=V_{\alpha}^{(2)}(x, y)+V_{\alpha}^{(2)}(y, z)$. Thus, for each $i \in\{1,2\}$, the condition $(\mathcal{J} 1)$ for family $\mathcal{V}^{(i)}$ holds.

Let $i \in\{1,2\}$ be arbitrary and fixed and let $\left(x_{m}: m \in \mathbb{N}\right)\left(\mathrm{y}_{m}: m \in \mathbb{N}\right)$ be arbitrary and fixed sequences in $X$ satisfying $\forall_{\alpha \in \mathcal{A}}\left\{\lim _{n \rightarrow \infty} \sup _{m>n} V_{\alpha}^{(i)}\left(x_{n}, x_{m}\right)=\lim _{m \rightarrow \infty} V_{\alpha}^{(i)}\left(x_{m}, y_{m}\right)=0\right\}$. Then, by Remark 3.2, we obtain that the conditions (2.1) and (2.2) for family $\mathcal{J}$ hold and, consequently, by $\forall_{\alpha \in \mathcal{A}}\left\{\lim _{m \rightarrow \infty} d_{\alpha}\left(x_{m}, y_{m}\right)=0\right\}, \forall_{\alpha \in \mathcal{A}}\left\{\lim _{m \rightarrow \infty} d_{\alpha}\left(x_{m}, y_{m}\right)=0\right\}$.This gives that $(\mathcal{J} 2)$ for family $\mathcal{V}^{(i)}$ holds.

We proved that, for each, $i \in\{1,2\}, \mathcal{V}^{(i)}$ is a $\mathcal{J}$-family.

Proposition 3.3 Let $X$ be a uniform space, let $\mathcal{J}=\left\{J_{\alpha}: X^{2} \rightarrow[0, \infty), \alpha \in \mathcal{A}\right\}$ be a $\mathcal{J}$-family and let $T: X \rightarrow X$.

(a) If $T$ and J satisfy (C1) or (C3), then $\left.\forall_{\alpha \in \mathcal{A}} \exists_{\lambda_{\alpha} \in[0,1)} \forall_{x \in X}\left\{\max J_{\alpha}\left(T(x), T^{[2]}(x)\right), J_{\alpha}\left(T^{[2]}(x), T(x)\right)\right\} \leq \lambda_{\alpha} \max \left\{J_{\alpha}(x, T(x)), J_{\alpha}(T(x), x)\right\}\right\}$.

(b) If $T$ and J satisfy (C2) or (C4), then $\forall_{\alpha \in \mathcal{A}_{\mathcal{A}}} \exists \lambda_{\lambda_{\alpha} \in[0,1)} \forall_{x \in X}\left\{J_{\alpha}\left(T^{[2]}(x), T(x)\right)+J_{\alpha}\left(T(x), T^{[2]}(x)\right) \leq \lambda_{\alpha}\left[J_{\alpha}(T(x), x)+J_{\alpha}(x, T(x))\right]\right\}$. 
Proof. (a) The proof will be broken into two steps.

STEP 1. If (C1) holds, then the assertion holds.

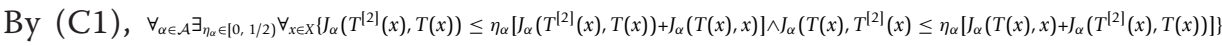
and, since $\forall_{\alpha \in \mathcal{A}}\left\{\eta_{\alpha} /\left(1-\eta_{\alpha}\right)<1\right\}$, we see that the first of these inequalities implies $\left.J_{\alpha}\left(T^{[2]}(x), T(x)\right) \leq \eta_{\alpha} /\left(1-\eta_{\alpha}\right)\right] J_{\alpha}(T(x), x) \leq J_{\alpha}(T(x), x) . \quad$ Hence $\forall_{\alpha \in \mathcal{A}} \exists_{\eta_{\alpha} \in[0,1 / 2)} \forall_{x \in X}\left\{\max \left\{J_{\alpha}\left(T(x), T^{[2]}(x)\right), J_{\alpha}\left(T^{[2]}(x), T(x)\right)\right\} \leq \eta_{\alpha}\left[J_{\alpha}\left(T^{[2]}(x), T(x)\right)+J_{\alpha}(T(x), x)\right] \leq 2 \eta_{\alpha} J_{\alpha}(T(x), x) \leq 2 \eta_{\alpha} \max \left\{J_{\alpha}(x, T(x)), J_{\alpha}(T(x), x)\right\}\right.$. Now, we see that $\forall_{\alpha \in \mathcal{A}}\left\{\lambda_{\alpha}=2 \eta_{\alpha}<1\right\}$.

STEP 2. If (C3) holds, then the assertion holds.

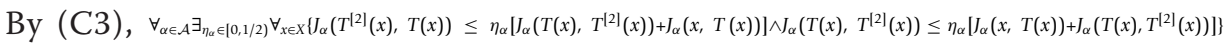
and, since $\forall_{\alpha \in \mathcal{A}}\left\{\eta_{\alpha} /\left(1-\eta_{\alpha}\right)<1\right\}$, we see that the second from these inequalities implies $J_{\alpha}\left(T(x), T^{[2]}(x)\right) \leq\left[\eta_{\alpha} /\left(1-\eta_{\alpha}\right)\right] J_{\alpha}(x, T(x)) \leq J_{\alpha}(x, T(x))$. Hence, we conclude that $\left.\left.\forall_{\alpha \in \mathcal{A}} \exists_{\eta_{\alpha}}[0,1 / 2) \forall_{x \in X}\left\{\max \mid J_{\alpha}\left(T^{[2]}(x), T(x)\right), J_{\alpha}\left(T(x), T^{[2 \mid}(x)\right)\right\} \leq \eta_{\alpha} \mid J_{\alpha}(x, T(x))+J_{\alpha}\left(T(x), T^{[2]}(x)\right)\right] \leq 2 \eta_{\alpha} J_{\alpha}(x, T(x)) \leq 2 \eta_{\alpha} \max \mid J_{\alpha}(x, T(x)), J_{\alpha}(T(x), x)\right\}$. It is clear that $\forall_{\alpha \in \mathcal{A}}\left\{\lambda_{\alpha}=2 \eta_{\alpha}<1\right\}$.

(b) The proof will be broken into two steps.

STEP 1. If (C2) holds, then the assertion holds.

By (C2), $\left.\quad \forall_{\alpha \in \mathcal{A}} \exists_{\eta_{\alpha}}[0,1 / 1 / 2) \forall_{x \in X}\left\{J_{\alpha}\left(T^{[2]}(x), T(x)\right) \leq \eta_{\alpha}\left(J_{\alpha}\left(T^{[2]}(x), T(x)\right)+J_{\alpha}(x, T(x))\right] \wedge_{\alpha} T(x), T^{|2|}(x)\right) \leq \eta_{\alpha}\left[J_{\alpha}(T(x), x)+J_{\alpha}\left(T(x), T^{[2]}(x)\right)\right]\right\}$. Hence, $\quad \forall_{\alpha \in \mathcal{A}} \exists_{\lambda_{\alpha} \in[0,1)} \forall_{x \in X}\left\{J_{\alpha}\left(T^{[2]}(x), T(x)\right) \leq \lambda_{\alpha} J_{\alpha}(x, T(x)) \wedge J_{\alpha}\left(T(x), T^{[2]}(x)\right) \leq \lambda_{\alpha} J_{\alpha}(T(x), x)\right\}$; here $\quad \forall_{\alpha \in \mathcal{A}}\left\{\lambda_{\alpha}=\eta_{\alpha} /\left(1-\eta_{\alpha}\right)\right\}$. From this, we conclude that $\forall_{\alpha \in \mathcal{A}} \exists_{\lambda_{\alpha} \in[0,1)} \forall_{x \in X}\left\{J_{\alpha}\left(T^{[2]}(x), T(x)\right)+J_{\alpha}\left(T(x), T^{[2]}(x)\right) \leq \lambda_{\alpha}\left[J_{\alpha}(T(x), x)+J_{\alpha}(x, T(x))\right]\right\}$.

STEP 2. If (C4) holds, then the assertion holds.

By (C4), $\left.\quad \forall_{\alpha \in \mathcal{A}} \exists_{\eta_{\alpha} \in[0,1 / 2)} \forall_{x \in X}\left\{J_{\alpha}\left(T^{[2]}(x), T(x)\right) \leq \eta_{\alpha}\left(J_{\alpha}\left(T(x), T^{[2]}(x)\right)+J_{\alpha}(T(x), x)\right] \wedge J_{\alpha} T(x), T^{[2]}(x)\right) \leq \eta_{\alpha}\left[J_{\alpha}(x, T(x))+J_{\alpha}\left(T^{[2]}(x), T(x)\right)\right]\right\}$.

This gives $\forall_{\alpha \in \mathcal{A}} \exists_{\eta_{\alpha} \in[0,1 / 2)} \forall_{x \in X}\left\{J_{\alpha}\left(T^{[2]}(x), T(x)\right) \leq \eta_{\alpha}^{2} /\left(1-\eta_{\alpha}^{2}\right) J_{\alpha}\left(x, T(x)+\eta_{\alpha} /\left(1-\eta_{\alpha}^{2}\right) J_{\alpha}(T(x), x) \wedge \wedge_{\alpha} T(x), T^{[2]}(x)\right) \leq \eta_{\alpha} /\left(1-\eta_{\alpha}^{2}\right) J_{\alpha}(x, T(x))+\eta_{\alpha}^{2} /\left(1-\eta_{\alpha}^{2}\right) J_{\alpha}(T(x), x)\right\}$. Hence, $\quad \forall_{\alpha \in \mathcal{A}} \exists_{\eta_{\alpha}}[0,1 / 2) \forall_{x \in X}\left[J_{\alpha}\left(T^{[2]}(x), T(x)\right)++_{\alpha} T(x), T^{[2]}(x)\right) \leq\left(\eta_{\alpha}+\eta_{\alpha}^{2}\right) /\left(1-n_{\alpha}^{2}\right)\left[J_{\alpha}(x, T(x))+J_{\alpha}(T(x), x)\right]=\eta_{\alpha} /\left(1-\eta_{\alpha}\right)\left[J_{\alpha} x,(T(x))+J_{\alpha}(T(x), x)\right]$. Since $\forall_{\alpha \in \mathcal{A}}\left\{\lambda_{\alpha}=\eta_{\alpha} /\left(1-\eta_{\alpha}\right)<1\right\}$ therefore

$\forall_{\alpha \in \mathcal{A}} \exists_{\lambda_{\alpha} \in[0,1)} \forall_{x \in X}\left\{J_{\alpha}\left(T^{[2]}(x), T(x)\right)+J_{\alpha} T(x), T^{[2]}(x)\right) \leq \lambda_{\alpha}\left[J_{\alpha}(x, T(x))+J_{\alpha}(T(x), x)\right]$.

Proposition 3.4 Let $X$ be a uniform space, let $\mathcal{J}=\left\{J_{\alpha}: X^{2} \rightarrow[0, \infty), \alpha \in \mathcal{A}\right\}$ be a $\mathcal{J}$-family and let $T: X \rightarrow X$. Assume that $T$ and $\mathcal{J}$ satisfy at least one of the conditions (C1)-(C4). Then:

(a) $\forall_{\alpha \in \mathcal{A}} \forall_{u^{0} \in X}\left\{\lim _{n \rightarrow \infty} \sup _{m>n} J_{\alpha}\left(u^{n}, u^{m}\right)=\lim _{n \rightarrow \infty} \sup _{m>n} J_{\alpha}\left(u^{m}, u^{n}\right)=0\right\}$.

(b) $\forall_{\alpha \in \mathcal{A}} \forall_{u^{0} \in X}\left\{\lim _{m \rightarrow \infty} J_{\alpha}\left(u^{m}, u^{m+1}\right)=\lim _{m \rightarrow \infty} J_{\alpha}\left(u^{m+1}, u^{m}\right)=0\right\}$.

(c) $\forall_{\alpha \in \mathcal{A}} \forall_{u^{0} \in X}\left\{\lim _{n \rightarrow \infty} \sup _{m>n} d_{\alpha}\left(u^{n}, u^{m}\right)=0\right\}$.

(d) If there exist $z \in X$ and $q \in \mathbb{N}$ such that $z=T^{[q]}(z)$, then $\operatorname{Fix}(T)=\{z\}$ and $\forall \forall_{\alpha \in \mathcal{A}}\left\{J_{\alpha}(z, z)=0\right\}$.

(e) If $v^{0}, w \in X$ satisfy (D1), then $\lim _{m \rightarrow \infty}{ }_{v}^{m}=w$.

(a) The proof will be broken into two steps.

STEP 1. If (C1) or (C3) holds, then the assertion holds.

There exist $\mathcal{J}$-families $\mathcal{W}^{(i)}=\left\{\mathcal{W}_{\alpha}^{(i)}, \alpha \in \mathcal{A}\right\}, i \in\{1,2\}$, such that

$$
\forall_{\alpha \in \mathcal{A}} \exists_{\lambda_{\alpha} \in[0,1)} \forall_{x \in X}\left\{W_{\alpha}^{(1)}\left(T(x), T^{[2]}(x)\right) \leq \lambda_{\alpha} W_{\alpha}^{(1)}(x, T(x))\right\}
$$

and

$$
\forall_{\alpha \in \mathcal{A}} \exists_{\lambda_{\alpha} \in[0,1)} \forall_{x \in x}\left\{W_{\alpha}^{(2)}\left(T^{[2]}(x), T(x)\right) \leq \lambda_{\alpha} W_{\alpha}^{(2)}(T(x), x)\right\} .
$$




$$
\begin{aligned}
& \text { Indeed, by Propositions 3.2(a) and 3.3(a), if } \\
& \forall_{\alpha \in \mathcal{A}} \forall_{x \in X}\left\{\phi_{\alpha}^{(1)}(x)=J_{\alpha}(T(x), x) \wedge \phi_{\alpha}^{(2)}(x)=J_{\alpha}(x, T(x))\right\} \text {, then the maps } \\
& W_{\alpha}^{(2)}(x, y)=\max \left\{\phi_{\alpha}^{(2)}(y), J_{\alpha}(x, y)\right\} \text { and } W_{\alpha}^{(2)}(x, y)=\max \left\{\phi_{\alpha}^{(2)}(y), J_{\alpha}(x, y)\right\}, x, y \in X \text {, }
\end{aligned}
$$
have properties (3.1)and (3.2), respectively.

Let $\alpha \in \mathcal{A}$ and $u^{0} \in X$ be arbitrary and fixed. By (3.1), using $(\mathcal{J} 1)$ for $\mathcal{J}$-family $\mathcal{W}^{(1)}, \quad$ if $m>n, \quad>\quad$ we get $W_{\alpha}^{(1)}\left(u^{n}, u^{m}\right) \leq \sum_{k=n}^{m-1} W_{\alpha}^{(1)}\left(u^{k}, u^{k+1}\right) \leq \sum_{k=n}^{m-1} \lambda_{\alpha}^{k} W_{\alpha}^{(1)}\left(u^{0}, u^{1}\right) \leq W_{\alpha}^{(1)}\left(u^{0}, u^{1}\right) \lambda_{\alpha}^{n} /\left(1-\lambda_{\alpha}\right)$. This gives $\lim _{n \rightarrow \infty} \sup _{m>n} W_{\alpha}^{(1)}\left(u^{n}, u^{m}\right)=0$ and, by Remark 3.2, we obtain $\forall_{\alpha \in \mathcal{A}} \forall_{u^{0} \in X}\left\{\lim _{n \rightarrow \infty} \sup _{m>n} J_{\alpha}\left(u^{n}, u^{m}\right)=0\right\}$.

If $\alpha \in \mathcal{A}$ and $u^{0} \in X$ are arbitrary and fixed, $m, n \in \mathbb{N}$ and $m>n$, then, by (3.2), using $\quad(\mathcal{J} 1) \quad$ for $\quad \mathcal{J}$-family $\quad \mathcal{W}^{(2),}$ we get $W_{\alpha}^{(2)}\left(u^{m}, u^{n}\right) \leq \sum_{k=n}^{m-1} W_{\alpha}^{(2)}\left(u^{k+1}, u^{k}\right) \leq \sum_{k=n}^{m-1} \lambda_{\alpha}^{k} W_{\alpha}^{(2)}\left(u^{1}, u^{0}\right) \leq W_{\alpha}^{(2)}\left(u^{1}, u^{0}\right) \lambda_{\alpha}^{n} /\left(1-\lambda_{\alpha}\right) \quad$ and $\lim _{n \rightarrow \infty} \sup _{m>n} W_{\alpha}^{(2)}\left(u^{m}, u^{n}\right)=0$. Hence, by Remark 3.2, $\forall_{\alpha \in \mathcal{A}} \forall_{u^{0} \in X}\left\{\lim _{n \rightarrow \infty} \sup _{m>n} J_{\alpha}\left(u^{m}, u^{n}\right)=0\right\}$.

STEP 2. If (C2) or (C4) holds, then the assertion holds.

There exist $\mathcal{J}$-families $\mathcal{V}^{(i)}=\left\{V_{\alpha^{\prime}}^{i} \alpha \in \mathcal{A}\right\}, i \in\{1,2\}$, such that

$$
\forall_{\alpha \in \mathcal{A}} \exists_{\lambda_{\alpha} \in[0,1)} \forall_{x \in X}\left\{V_{\alpha}^{(1)}\left(T(x), T^{[2]}(x)\right) \leq \lambda_{\alpha} V_{\alpha}^{(1)}(x, T(x))\right\}
$$

and

$$
\forall_{\alpha \in \mathcal{A}} \exists_{\lambda_{\alpha} \in[0,1)} \forall_{x \in X}\left\{V_{\alpha}^{(2)}\left(T^{[2]}(x), T(x)\right) \leq \lambda_{\alpha} V_{\alpha}^{(2)}(T(x), x)\right\} .
$$

Indeed, by Propositions 3.2(b) and 3.3(b), we have that if $\forall_{\alpha \in \mathcal{A}} \forall_{x \in X}\left\{\phi_{\alpha}^{(1)}(x)=J_{\alpha}(T(x), x) \wedge \phi_{\alpha}^{(2)}(x)=J_{\alpha}(x, T(x))\right\}$, then the maps $V_{\alpha}^{(1)}(x, y)=\phi_{\alpha}^{(1)}(x)+J_{\alpha}(x, y)$ and $V_{\alpha}^{(2)}(x, y)=\phi_{\alpha}^{(2)}(y)+J_{\alpha}(x, y), x, y \in X$, have the above properties.

Let $\alpha \in \mathcal{A}$ and $u^{0} \in X$ are arbitrary and fixed. Then, by (3.3), using (J1) for $\mathcal{V}^{(1)}$-family $\mathcal{V}^{(1)}, \quad$ if $m>n$, we have $V_{\alpha}^{(1)}\left(u^{n}, u^{m}\right) \leq \sum_{k=n}^{m-1} V_{\alpha}^{(1)}\left(u^{k}, u^{k+1}\right) \leq \sum_{k=n}^{m-1} \lambda_{\alpha}^{k} V_{\alpha}^{(1)}\left(u^{0}, u^{1}\right) \leq V_{\alpha}^{(1)}\left(u^{0}, u^{1}\right) \lambda_{\alpha}^{n} /\left(1-\lambda_{\alpha}\right) . \quad$ consequently, $\quad \lim _{n \rightarrow \infty} \sup _{m>n} V_{\alpha}^{(1)}\left(u^{n}, u^{m}\right)=0$. By Remark 3.2, this gives $\forall_{\alpha \in \mathcal{A}} \forall_{u^{0} \in X}\left\{\lim _{n \rightarrow \infty} \sup _{m>n} J_{\alpha}\left(u^{n}, u^{m}\right)=0\right\}$.

If $\alpha \in \mathcal{A}$ and $u^{0} \in X$ are arbitrary and fixed, $m, n \in \mathbb{N}$ and $m>n$, then, by (3.4), using $(\mathcal{J} 1) \quad$ for $\quad \mathcal{J}$-family $\mathcal{V}^{(2),}$ we have $V_{\alpha}^{(2)}\left(u^{m}, u^{n}\right) \leq \sum_{k=n}^{m-1} V_{\alpha}^{(2)}\left(u^{k+1}, u^{k}\right) \leq \sum_{k=n}^{m-1} \lambda_{\alpha}^{k} V_{\alpha}^{(2)}\left(u^{1}, u^{0}\right) \leq V_{\alpha}^{(2)}\left(u^{1}, u^{0}\right) \lambda_{\alpha}^{n}\left(1-\lambda_{\alpha}\right)$. Hence, we obtain that $\lim _{n \rightarrow \infty} \sup _{m>n} V_{\alpha}^{(2)}\left(u^{m}, u^{n}\right)=0$. By Remark 3.2, this gives $\forall_{\alpha \in \mathcal{A}} \forall_{u^{0} \in X}\left\{\lim _{n \rightarrow \infty} \sup _{m>n} J_{\alpha}\left(u^{m}, u^{n}\right)=0\right\}$.

(b) This is a consequence of (a) since $\forall_{\alpha \in \mathcal{A}} \forall_{u^{0} \in X} \forall_{n \in \mathbb{N}}\left\{J_{\alpha}\left(u^{n}, u^{n+1}\right) \leq \sup _{m>n} J_{\alpha}\left(u^{n}, u^{m}\right) \wedge J_{\alpha}\left(u^{n+1}, u^{n}\right) \leq \sup _{m>n} J_{\alpha}\left(u^{m}, u^{n}\right)\right\}$. 
(c) Let $u^{0} \in X$ be arbitrary and fixed. By (a),

$$
\forall \forall_{\alpha \in \mathcal{A}}\left\{\lim _{n \rightarrow \infty} \sup _{m>n} J_{\alpha}\left(u^{n}, u^{m}\right)=0\right\}
$$

which implies $\forall_{\alpha \in \mathcal{A}} \forall_{\varepsilon>0} \exists_{n_{1}=n_{1}(\alpha, \varepsilon) \in \mathbb{N}} \forall_{n>n_{1}}\left\{\sup \left\{J_{\alpha}\left(u^{n}, u^{m}\right): m>n\right\}<\varepsilon\right\}$ and, in particular,

$$
\forall_{\alpha \in \mathcal{A}} \forall_{\in>0} \exists_{n_{1}=n_{1}(\alpha, \epsilon) \in \mathbb{N}} \forall_{n>n_{1}} \forall_{s \in \mathbb{N}}\left\{J_{\alpha}\left(u^{n}, u^{s+n}\right)<\varepsilon\right\} .
$$

Let now $i_{0}, j_{0} \in \mathbb{N}, i_{0}>j_{0}$, be arbitrary and fixed. If we define

$$
x_{m}=u^{i_{0}+m} \text { and } y_{m}=u^{j_{0}+m} \quad \text { for } m \in \mathbb{N},
$$

then (3.6) gives

$$
\forall_{\alpha \in \mathcal{A}}\left\{\lim _{m \rightarrow \infty} J_{\alpha}\left(u^{m}, x_{m}\right)=\lim _{m \rightarrow \infty} J_{\alpha}\left(u^{m}, y_{m}\right)=0\right\} .
$$

Therefore, by (3.5), (3.8) and (J 2),

$$
\forall_{\alpha \in \mathcal{A}}\left\{\lim _{m \rightarrow \infty} d_{\alpha}\left(u^{m}, x_{m}\right)=\lim _{m \rightarrow \infty} d_{\alpha}\left(u^{m}, y_{m}\right)=0\right\} .
$$

From (3.7) and (3.9), we then claim that

$$
\forall_{\alpha \in \mathcal{A}} \forall_{\varepsilon>0} \exists_{n_{2}=n_{2}(\alpha, \varepsilon) \in \mathbb{N}} \forall_{m>n_{2}}\left\{d_{\alpha}\left(u^{m}, u^{i_{0}+m}\right)<\varepsilon / 2\right\}
$$

and

$$
\forall_{\alpha \in \mathcal{A}} \forall_{\varepsilon>0} \exists_{n_{3}=n_{3}(\alpha, \varepsilon) \in \mathbb{N}} \forall_{m>n_{3}}\left\{d_{\alpha}\left(u^{m}, u^{j_{0}+m}\right)<\varepsilon / 2\right\} .
$$

Let now $\alpha_{0} \in \mathcal{A}$ and $\varepsilon_{0}>0$ be arbitrary and fixed, let $n_{0}=\max \left\{n_{2}\left(\alpha_{0}, \varepsilon_{0}\right), n_{3}\left(\alpha_{0}\right.\right.$, $\left.\left.\varepsilon_{0}\right)\right\}+1$ and let $k, l \in \mathbb{N}$ be arbitrary and fixed such that $k>l>n_{0}$. Then, $k=i_{0}+n_{0}$ and $l=j_{0}+n_{0}$ for some $i_{0}, j_{0} \in \mathbb{N}$ such that $i_{0}>j_{0}$ and, using (3.10) and (3.11), we get $d_{\alpha_{0}}\left(u^{k}, u^{l}\right)=d_{\alpha_{0}}\left(u^{i_{0}+n_{0}}, u^{j_{0}+n_{0}}\right) \leq d_{\alpha_{0}}\left(u^{n_{0}}, u^{i_{0}+n_{0}}\right)+d_{\alpha_{0}}\left(u^{n_{0}}, u^{j_{0}+n_{0}}\right)<\varepsilon_{0} / 2+\varepsilon_{0} / 2=\varepsilon_{0}$. Hence, we conclude that $\forall_{\alpha \in \mathcal{A}} \forall_{\varepsilon>0} \exists_{n_{0}=n_{0}(\alpha, \varepsilon) \in \mathbb{N}} \forall_{k, l \in \mathbb{N}, k>l>n_{0}}\left\{d_{\alpha}\left(u^{k}, u^{l}\right)<\varepsilon\right\}$.

(d) The proof will be broken into two steps.

STEP 1. If (C1) or (C3) holds, then the assertions hold.

Let $z \in$ Fix $\left(T^{[q]}\right)$ for some $z \in X$ and $q \in \mathbb{N}$. First, we prove that if $\mathcal{W}^{(1)}$ is $\mathcal{J}$-family defined in the proof of (a), then

$$
\forall_{\alpha \in A}\left\{W_{\alpha}^{(1)}(z, T(z))=0\right\} .
$$

Otherwise, we have $\exists_{\alpha_{0} \in \mathcal{A}}\left\{W_{\alpha_{0}}^{(1)}(z, T(z))>0\right\}$. Then, by (3.1), since $z=T^{[q]}(z)=$ $T^{[2 q]}=T^{[2 q]}(z)$, there exists $\lambda_{\alpha_{0}} \in[0,1)$, such that $W_{\alpha_{0}}^{(1)}(z, T(z))=W_{\alpha_{0}}^{(1)}\left(T^{[2 q]}(z)\right.$, $\left.T^{[2]}\left(T^{[2 q-1]}(z)\right)\right) \leq \lambda_{\alpha_{0}} W_{\alpha_{0}}^{(1)}\left(T\left(T^{[2 q-2]}(z)\right), \quad T^{[2]}\left(T^{[2 q-1]}(z)\right)\right) \leq \lambda_{\alpha_{0}} W_{\alpha_{0}}^{(1)}\left(T\left(T^{[2 q-2]}(z)\right)\right.$, $\left.T\left(T^{[2 q-2]}(z)\right)\right) \leq \cdots \leq \lambda_{\alpha_{0}}^{2 q} W_{\alpha_{0}}^{(1)}(z, T(z))<W_{\alpha_{0}}^{(1)}(z, T(z))$, $\left.T\left(T^{[2 q-2]}(z)\right)\right) \leq \cdots \leq \lambda_{\alpha_{0}}^{2 q} W_{\alpha_{0}}^{(1)}(z, T(z))<W_{\alpha_{0}}^{(1)}(z, T(z))$, which is absurd. Therefore, (3.12) is satisfied. 
Next, we see that

$$
\forall_{\alpha \in \mathcal{A}}\left\{W_{\alpha}^{(1)}(T(z), z)=0\right\}
$$

Otherwise, $\exists_{\alpha_{0} \in A}\left\{W_{\alpha_{0}}^{(1)}(T(z), z)>0\right\}$ and, since $z=T^{[q]}(z)=T^{[2 q]}(z)$ and if $q+1$ $<2 q$, then by (3.1) and (3.12), for some $\lambda_{\alpha_{0}} \in[0,1)$, we get 0 $<0<W_{\alpha_{0}}^{(1)}(T(z), z)=W_{\alpha_{0}}^{(1)}\left(T\left(T^{[q]}(z)\right), \quad T^{[2 q]}(z)\right)=W_{\alpha_{0}}^{(1)}\left(T^{[q+1]}(z)\right.$, $\left.T^{[2 q]}(z)\right) \leq \sum_{i=q+1}^{2 q-1} \lambda_{\alpha_{0}}^{i} W_{\alpha_{0}}^{(1)}(z, T(z)) \leq\left[\lambda_{\alpha_{0}}^{q+1} /\left(1-\lambda_{\alpha_{0}}\right)\right] W_{\alpha_{0}}^{(1)}(z, T(z))=0, \quad$ which is absurd. If $q+1=2 q$, i.e. $q=1$, then $z=T(z)=T^{2}(z)$ and, by (3.1) and (3.12), $0<W_{\alpha_{0}}^{(1)}(T(z), z)=W_{\alpha_{0}}^{(1)}\left(T(z), T^{[2]}(z)\right) \leq \lambda_{\alpha_{0}} W_{\alpha_{0}}^{(1)}(z, T(z))=0$, which is absurd. Therefore, (3.13) holds.

Now, using Remark 3.2, we see that (3.12) and (3.13) gives

$$
\forall_{\alpha \in A}\left\{J_{\alpha}(z, T(z))=J_{\alpha}(T(z), z)=0\right\},
$$

which, by Remark 3.1, implies that $z \in$ Fix $(T)$.

By $(\mathcal{J} 1)$ and (3.14), we obtain $\forall_{\alpha \in \mathcal{A}}\left\{J_{\alpha}(z, z) \leq J_{\alpha}(z, T(z))+J_{\alpha}(T(z), z)=0\right\}$.

We show that $z$ is a unique fixed point of $T$. Otherwise, there exist $a, b \in$ Fix $(T)$ such that $a \neq b$. Then, using above for $q=1$, we obtain $\forall_{\alpha \in \mathcal{A}}\left\{J_{\alpha}(a, T(a))=J_{\alpha}(T(a), a)=0\right\}$ and $\forall_{\alpha \in A}\left\{J_{\alpha}(b, T(b))=J_{\alpha}(T(b), b)=0\right\}$. Hence, if (C1) holds, then for each $\alpha \in \mathcal{A}$, by $(\mathrm{C} 1), J_{\alpha}(a, b)=J_{\alpha}(T(a), T(b)) \leq \eta_{\alpha}\left[J_{\alpha}(T(a), a)+\right.$ $\left.J_{\alpha}(T(b), b)\right]=0$ and $J_{\alpha}(b, a)=J_{\alpha}(T(b), T(a)) \leq \eta_{\alpha}\left[J_{\alpha}(T(b), b)+J_{\alpha}(T(a), a)\right]=0$ where $\eta_{\alpha} \in[0,1 / 2)$. Hence, $\forall_{\alpha \in \mathcal{A}}\left\{J_{\alpha}(a, b)=J_{\alpha}(b, a)=0\right\}$. By Remark 3.1, this implies $a=b$. Contradiction. Similarly, if (C3) holds, then, for each $\alpha \in \mathcal{A}$, by (C3), $J_{\alpha}(a, b)=$ $J_{\alpha}(T(a), T(b)) \leq \eta_{\alpha}\left[J_{\alpha}(a, T(a))+J_{\alpha}(b, T(b))\right]=0$ and $J_{\alpha}(b, a)=J_{\alpha}(T(b), T(a)) \leq$ $\eta_{\alpha}\left[J_{\alpha}(b, T(b))+J_{\alpha}(a, T(a))\right]=0$ where $\eta_{\alpha} \in[0,1 / 2)$. Thus, $\forall_{\alpha \in \mathcal{A}}\left\{J_{\alpha}(a, b)=J_{\alpha}(b, a)=0\right\}$ which, by Remark 3.1, implies $a=b$. Contradiction.

STEP 2. If (C2) or (C4) holds, then the assertions hold.

Let $z \in \operatorname{Fix}\left(T^{[q]}\right)$ for some $z \in X$ and $q \in \mathbb{N}$. We prove that if $\mathcal{V}^{(1)}$ is $\mathcal{J}$-family defined in the proof of (a), then

$$
\forall_{\alpha \in \mathcal{A}}\left\{V_{\alpha}^{(1)}(z, T(z))=0\right\}
$$

Otherwise, we have $\exists_{\alpha_{0} \in \mathcal{A}}\left\{V_{\alpha_{0}}^{(1)}(z, T(z))>0\right\}$ and, consequently, by (3.3), since $z=T$ ${ }^{[q]}(z)=T^{[2 q]}(z)$, we get, for some $\lambda_{\alpha_{0}} \in[0,1), V_{\alpha_{0}}^{(1)}(z, T(z))=V_{\alpha_{0}}^{(1)}\left(T^{[2 q]}(z)\right.$, $\left.T^{[2]}\left(T^{[2 q-1]}(z)\right)\right) \leq \lambda_{\alpha_{0}} V_{\alpha_{0}}^{(1)}\left(T\left(T^{[2 q-2]}(z)\right), \quad T^{[2]}\left(T^{[2 q-1]}(z)\right)\right) \leq \lambda_{\alpha_{0}} V_{\alpha_{0}}^{(1)}\left(T\left(T^{[2 q-2]}(z)\right)\right.$, $\left.T\left(T^{[2 q-2]}(z)\right)\right) \leq \cdots \leq \lambda_{\alpha_{0}}^{2 q} V_{\alpha_{0}}^{(1)}(z, T(z))<V_{\alpha_{0}}^{(1)}(z, T(z))$, $\left.T\left(T^{[2 q-2]}(z)\right)\right) \leq \cdots \leq \lambda_{\alpha_{0}}^{2 q} V_{\alpha_{0}}^{(1)}(z, T(z))<V_{\alpha_{0}}^{(1)}(z, T(z))$, which is absurd. Therefore, (3.15) holds.

Next, we prove that

$$
\forall_{\alpha \in \mathcal{A}}\left\{V_{\alpha}^{(1)}(T(z), z)=0\right\}
$$

Otherwise, $\exists_{\alpha_{0} \in \mathcal{A}}\left\{V_{\alpha_{0}}^{(1)}(T(z), z)>0\right\}$ and, since $z=T^{[q]}(z)=T^{[2 q]}(z)$, if $q+1<2 q$, then, by (3.3) and (3.15), for some $\lambda_{\alpha_{0}} \in[0,1)$, we have 


$$
\begin{aligned}
& \left.T^{[2 q]}(z)\right) \leq \sum_{i=q+1}^{2 q-1} \lambda_{\alpha_{0}}^{i} V_{\alpha_{0}}^{(1)}(z, T(z)) \leq\left[\lambda_{\alpha_{0}}^{q+1} /\left(1-\lambda_{\alpha_{0}}\right)\right] V_{\alpha_{0}}^{(1)}(z, T(z))=0, \\
& \left.T^{[2 q]}(z)\right) \leq \sum_{i=q+1}^{2 q-1} \lambda_{\alpha_{0}}^{i} V_{\alpha_{0}}^{(1)}(z, T(z)) \leq\left[\lambda_{\alpha_{0}}^{q+1} /\left(1-\lambda_{\alpha_{0}}\right)\right] V_{\alpha_{0}}^{(1)}(z, T(z))=0, \\
& \left.T^{[2 q]}(z)\right) \leq \sum_{i=q+1}^{2 q-1} \lambda_{\alpha_{0}}^{i} V_{\alpha_{0}}^{(1)}(z, T(z)) \leq\left[\lambda_{\alpha_{0}}^{q+1} /\left(1-\lambda_{\alpha_{0}}\right)\right] V_{\alpha_{0}}^{(1)}(z, T(z))=0, \quad \text { which } \quad \text { is }
\end{aligned}
$$

absurd. If $q+1=2 q$, i.e. $q=1$, then $z T(z)=T^{2}(z)$ and, by (3.3) and (3.15), $\left.\left.T^{[2]}(z)\right) \leq \lambda_{\alpha_{0}} V_{\alpha_{0}}^{(1)}(z, T(z))=0, \quad T^{[2]}(z)\right) \leq \lambda_{\alpha_{0}} V_{\alpha_{0}}^{(1)}(z, T(z))=0$, which is absurd.

Therefore, (3.16) holds.

By Remark 3.2, (3.15) and (3.16) implies

$$
\forall_{\alpha \in \mathcal{A}}\left\{J_{\alpha}(z, T(z))=J_{\alpha}(T(z), z)=0\right\},
$$

which, by Remark 3.1, gives $z \in$ Fix $(T)$.

Now, by $(\mathcal{J} 1)$ and (3.17), we obtain $\forall_{\alpha \in \mathcal{A}}\left\{J_{\alpha}(z, z) \leq J_{\alpha}(z, T(z))+J_{\alpha}(T(z), z)=0\right\}$.

We show that $z$ is a unique fixed point of $T$. Otherwise, there exist $a, b \in F i x(T)$ such that $a \neq b$. Then, by above considerations for $q=1$, we get $\forall_{\alpha \in \mathcal{A}}\left\{J_{\alpha}(a, T(a))=J_{\alpha}(T(a), a)=0\right\}$ and $\forall_{\alpha \in \mathcal{A}}\left\{J_{\alpha}(b, T(b))=J_{\alpha}(T(b), b)=0\right\}$. Hence, if (C2) holds, then for each $\alpha \in \mathcal{A}$, by (C2), $J_{\alpha}(a, b)=J_{\alpha}(T(a), T(b)) \leq \alpha\left[J_{\alpha}(T(a), a)\right.$ $\left.+J_{\alpha}(b, T(b))\right]=0$ and $J_{\alpha}(b, a)=J_{\alpha}(T(b), T(a)) \leq \eta_{\alpha}\left[J_{\alpha}(T(b), b)+J_{\alpha}(a, T(a))\right]=$ 0 where $\eta_{\alpha} \in[0,1 / 2)$. Therefore, $\forall_{\alpha \in \mathcal{A}}\left\{J_{\alpha}(a, b)=J_{\alpha}(b, a)=0\right\}$. Hence, by Remark 3.1, we get $a=b$, which is impossible. Similarly, if (C4) holds, then, for each $\alpha \in \mathcal{A}$, by (C4), $J_{\alpha}(a, b)=J_{\alpha}(T(a), T(b)) \leq \eta_{\alpha}\left[J_{\alpha}(a, T(a))+J_{\alpha}(T(b), b)\right]=0$ and $J_{\alpha}(b, a)=$ $\left.J_{\alpha}(T(b), T(a)) \leq \eta_{\alpha} J_{\alpha}(b, T(b))+J_{\alpha}(T(a), a)\right]=0$ where $\eta_{\alpha} \in[0,1 / 2)$. Therefore $\forall_{\alpha \in \mathcal{A}}\left\{J_{\alpha}(a, b)=J_{\alpha}(b, a)=0\right\}$ and, by Remark 3.1, we get $a=b$, which is impossible.

(e) Indeed, by (a), we have $\forall_{\alpha \in \mathcal{A}}\left\{\lim _{m \rightarrow \infty} \sup _{m>n} J_{\alpha}\left(v^{n}, v^{m}\right)=0\right\}$. Next, by (D1), $\forall_{\alpha \in \mathcal{A}}\left\{\lim _{m \rightarrow \infty} J_{\alpha}\left(v^{m}, w\right)=0\right\}$. Hence, defining $x_{m}=v^{m}$ and $y_{m}=w$ for $m \in \mathbb{N}$, we conclude that (2.1) and (2.2) hold for sequences $\left(x_{m}: m \in \mathbb{N}\right)$ and $\left(y_{m}: m \in \mathbb{N}\right)$ in $X$. Therefore, by (J2), we get (2.3) which implies $\forall_{\alpha \in \mathcal{A}}\left\{\lim _{m \rightarrow \infty} d_{\alpha}\left(v^{m}, w\right)=0\right\}$.

\section{Proof of Theorem 2.1}

The proof will be broken into 11 steps.

STEP 1. If $v^{0}, \quad w \quad X \quad X \quad$ satisfy (D1), then $\forall_{\alpha \in A}\left\{\lim _{m \rightarrow \infty} J_{\alpha}\left(v^{m}, v^{m+1}\right)=\lim _{m \rightarrow \infty} J_{\alpha}\left(v^{m+1}, v^{m}\right)=0\right\}$. This follows from Proposition 3.4(b).

STEP 2. If $v^{0}, w \in X$ satisfy (D1), then $\lim _{m \rightarrow \infty} v^{m}=w$. This follows from Proposition 3.4(e).

STEP 3. If $v^{0}, w \in X$ satisfy (D1), then $\forall_{\alpha \in \mathcal{A}}\left\{J_{\alpha}(T(w), w)=0\right\}$.

Indeed, by $(\mathcal{J} 1)$ and $(\mathrm{C} 1), \quad \forall_{\alpha \in \mathcal{A}} \exists_{\eta_{\alpha} \in[0,1 / 2)} \forall_{m \in \mathbb{N}}\left\{J_{\alpha}(T(w), w) \leq J_{\alpha}(T(w)\right.$, $\left.\left.T\left(v^{m}\right)\right)+J_{\alpha}\left(T\left(v^{m}\right), w\right) \leq \eta_{\alpha}\left[J_{\alpha}(T(w), w)+J_{\alpha}\left(v^{m+1}, v^{m}\right)\right]+J_{\alpha}\left(v^{m+1}, w\right)\right\}$. Hence, by Step 1 and (D1), we obtain $\forall_{\alpha \in \mathcal{A}} \exists_{\eta_{\alpha} \in[0,1 / 2)}\left\{\lim _{m \rightarrow \infty} J_{\alpha}(T(w), w) \leq \lim _{m \rightarrow \infty}\left\{\eta_{\alpha}\left[J_{\alpha}(T(w), w)+J_{\alpha}\left(v^{m+1}, v^{m}\right)\right]+J_{\alpha}\left(v^{m+1}, w\right)\right\}=\eta_{\alpha} J_{\alpha}(T(w), w)\right\} . \quad$ Thus, $\forall_{\alpha \in A} \exists_{\eta_{\alpha} \in[0,1 / 2)}\left\{J_{\alpha}(T(w), w) \leq \eta_{\alpha} J_{\alpha}(T(w), w)\right\}$, so, since $\forall_{\alpha \in \mathcal{A}}\left\{\eta_{\alpha} \in[0,1 / 2)\right\}$, we get $\forall_{\alpha \in \mathcal{A}}\left\{J_{\alpha}(T(w), w)=0\right\}$.

STEP 4. If $v^{0}, w \in X$ satisfy (D1), then $T(w) \in \operatorname{Fix}(T)$. 
Indeed, by (C1) and Step 3, we have that $\forall_{\alpha \in \mathcal{A}} \exists_{\eta_{\alpha} \in[0,1 / 2)}\left\{J_{\alpha}\left(T^{[2]}(w), T(w)\right) \leq \eta_{\alpha}\left[J_{\alpha}\left(T^{[2]}(w), T(w)\right)+J_{\alpha}(T(w), w)\right]=\eta_{\alpha} J_{\alpha}\left(T^{[2]}(w), T(w)\right)\right\}$. Hence,

$$
\forall_{\alpha \in \mathcal{A}}\left\{J_{\alpha}\left(T^{[2]}(w), T(w)\right)=0\right\} .
$$

On the other hand, by (C1), $\forall_{\alpha \in \mathcal{A}} \exists_{\eta_{\alpha} \in[0,1 / 2)}\left\{J_{\alpha}\left(T(w), T^{[2]}(w)\right) \leq \eta_{\alpha}\left[J_{\alpha}(T(w), w)+J_{\alpha}\left(T^{[2]}(w), T(w)\right)\right]\right\}$. Hence, by Step 3 and (4.1),

$$
\forall_{\alpha \in \mathcal{A}}\left\{J_{\alpha}\left(T(w), T^{[2]}(w)\right)=0\right\}
$$

Now, by (4.1), (4.2) and Remark 3.1, we conclude that $T(w)=T^{2}(w)=T(T(w))$, i.e. $T$ $(w) \in \operatorname{Fix}(T)$ is satisfied.

STEP 5. If $v^{0}, w \in X$ satisfy (D1), then $\forall_{\alpha \in \mathcal{A}}\left\{\lim _{m \rightarrow \infty} J_{\alpha}\left(v^{m}, T(w)\right)=0\right\}$.

Indeed, by (C1) and Step 3,

$\forall_{\alpha \in \mathcal{A}} \exists_{\eta_{\alpha} \in[0,1 / 2)}\left\{J_{\alpha}\left(v^{m}, T(w)\right) \leq \eta_{\alpha}\left[J_{\alpha}\left(v^{m}, v^{m-1}\right)+J_{\alpha}(T(w), w)\right]=\eta_{\alpha} J_{\alpha}\left(v^{m}, v^{m-1}\right)\right\}$. Hence, by

Step 1, we obtain that $\forall_{\alpha \in \mathcal{A}}\left\{\lim _{m \rightarrow \infty} J_{\alpha}\left(v^{m}, T(w)\right)=0\right\}$.

STEP 6. If $v^{0}, w \in X$ satisfy (D1), then $\lim _{m \rightarrow \infty} v^{m}=T(w)$.

Indeed, by (C1) and Proposition 3.4(a), in particular, $\forall_{\alpha \in A}\left\{\lim _{n \rightarrow \infty} \sup _{m>n} J_{\alpha}\left(v^{n}, v^{m}\right)=0\right\}$. Next, by Step 5, $\forall_{\alpha \in \mathcal{A}}\left\{\lim _{m \rightarrow \infty} J_{\alpha}\left(v^{m}, T(w)\right)=0\right\}$. Hence, defining $x_{m}=v^{m}$ and $y_{m}=T(w)$ for $m \in \mathbb{N}$, we conclude that for sequences $\left(x_{m}: m \in \mathbb{N}\right)$ and $\left(y_{m}: m \in \mathbb{N}\right)$ in $X$ the conditions (2.1) and (2.2) hold. Consequently, by $(\mathcal{J} 2)$, we get (2.3), which implies

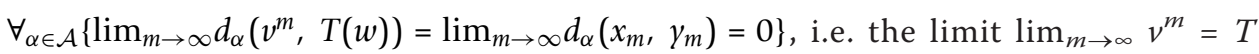
(w) holds.

STEP 7. If $v^{0}, w \in X$ satisfy (D1), then $T(w)=w$ and $\forall_{\alpha \in \mathcal{A}}\left\{J_{\alpha}(w, w)=0\right\}$.

Since $X$ is Hausdorff, thus $T(w)=w$ is a consequence of Steps 2 and 6 .

Next, by (C1) and Step 3, we obtain $\forall_{\alpha \in \mathcal{A}} \exists_{\eta_{\alpha} \in[0,1 / 2)}\left\{J_{\alpha}(w, w)=J_{\alpha}(T(w), T(w)) \leq \eta_{\alpha}\left[J_{\alpha}(T(w), w)+J_{\alpha}(T(w), w)\right]=0\right\}, \quad$ i.e.

$\forall_{\alpha \in \mathcal{A}}\left\{J_{\alpha}(w, w)=0\right\}$ holds.

STEP 8. If $v^{0}, w \in X$ satisfy (D1), then $\forall_{\alpha \in \mathcal{A}} \forall_{u^{0} \in X}\left\{\lim _{m \rightarrow \infty} J_{\alpha}\left(u^{m}, w\right)=0\right\}$.

By $(\mathcal{J} 1), \quad(\mathrm{C} 1)$, Proposition 3.4(b), Step 1 and (D1), we obtain $\forall_{\alpha \in \mathcal{A}} \exists_{\eta_{a}}[0,1 / 2) \forall_{u^{0} \in X}\left\{\lim _{m \rightarrow \infty} J_{\alpha}\left(u^{m}, w\right) \leq \lim _{m \rightarrow \infty}\left[J_{\alpha}\left(u^{m}, v^{m}\right)+J_{\alpha}\left(v^{m}, w\right)\right] \leq \eta_{\alpha} \lim _{m \rightarrow \infty}\left[J_{\alpha}\left(u^{m}, u^{m-1}\right)+J_{\alpha}\left(v^{m}, v^{m-1}\right)\right]+\lim _{m \rightarrow \infty} J_{\alpha}\left(v^{m}, w\right)=0\right\}$.

STEP 9. If $v^{0}, w \in X$ satisfy (D1), then $\forall_{\alpha \in \mathcal{A}} \forall_{u^{0} \in X}\left\{\lim _{m \rightarrow \infty} d_{\alpha}\left(u^{m}, w\right)=0\right\}$.

Indeed, by (C1), Proposition 3.4(a) and Step 8, $\forall_{\alpha \in \mathcal{A}} \forall_{u^{0} \in X}\left\{\lim _{n \rightarrow \infty} \sup _{m>n} J_{\alpha}\left(u^{n}, u^{m}\right)=0\right\} \quad$ and $\quad \forall_{\alpha \in \mathcal{A}} \forall_{u^{0} \in X}\left\{\lim _{m \rightarrow \infty} J_{\alpha}\left(u^{m}, w\right)=0\right\}$. Defining $x_{m}=u_{m}$ and $y_{m}=w$ for $m \in \mathbb{N}$, we conclude that for sequences $\left(x_{m}: m \in\right.$ $\mathbb{N})$ and $\left(y_{m}: m \in \mathbb{N}\right)$ in $X$ the conditions (2.1) and (2.2) hold. Hence, by (J2), we get (2.3) which implies $\forall_{\alpha \in \mathcal{A}} \forall_{u^{0} \in X}\left\{\lim _{m \rightarrow \infty} d_{\alpha}\left(u^{m}, w\right)=0\right\}$.

STEP 10. If $v^{0}, w \in X$ satisfy (D1), then Fix $(T)=\{w\}$.

Indeed, by (C1), Step 7 and Proposition 3.4(d) (for $q=1$ ), we get that Fix $(T)=\{w\}$.

STEP 11. The assertions (a)-(c) are satisfied.

This is a consequence of Steps 10, 9 and 7.

\section{Proof of Theorem 2.2}

The proof will be broken into seven steps. 
STEP 1. If $v^{0}, \quad w \in X \quad$ satisfy $\quad$ (D2), then $\forall_{\alpha \in \mathcal{A}}\left\{\lim _{m \rightarrow \infty} J_{\alpha}\left(v^{m}, v^{m+1}\right)=\lim _{m \rightarrow \infty} J_{\alpha}\left(v^{m+1}, v^{m}\right)=0\right\}$. This follows from Proposition 3.4(b).

STEP 2. If $v^{0}, w \in X$ satisfy (D2), then $\lim _{m \rightarrow \infty} v^{m}=w$. This follows from Proposition $3.4(\mathrm{e})$.

STEP 3. If $v^{0}, w \in X$ satisfy (D2), then $T(w)=w$ and $\forall_{\alpha \in \mathcal{A}}\left\{J_{\alpha}(w, w)=0\right\}$.

Indeed, we consider three cases:

Case 1. If (C2) holds, then by $(\mathcal{J} 1)$ and $(\mathrm{C} 2)$, we have $\forall_{\alpha \in \mathcal{A}} \exists_{\eta_{\alpha} \in[0,1 / 2)} \forall_{m \in \mathbb{N}}\left\{J_{\alpha}(w, T(w)) \leq J_{\alpha}\left(w, T\left(v^{m}\right)\right)+J_{\alpha}\left(T\left(v^{m}\right), T(w)\right) \leq J_{\alpha}\left(w, v^{m+1}\right)+\eta_{\alpha}\left[J_{\alpha}\left(v^{m+1}, v^{m}\right)+J_{\alpha}(w, T(w))\right]\right\}$. Consequently, by (D2) and Step $\forall_{\alpha \in \mathcal{A}} \exists_{\eta_{\alpha} \in[0,1 / 2)}\left\{\lim _{m \rightarrow \infty} J_{\alpha}(w, T(w)) \leq \lim _{m \rightarrow \infty}\left[J_{\alpha}\left(w, v^{m+1}\right)+\eta_{\alpha} J_{\alpha}\left(v^{m+1}, v^{m}\right)+\eta_{\alpha} J_{\alpha}(w, T(w))\right]=\eta_{\alpha} J_{\alpha}(w, T(w))\right\}, \quad$ i.e. $\forall_{\alpha \in \mathcal{A}} \exists_{\eta_{\alpha} \in[0,1 / 2)}\left\{J_{\alpha}(w, T(w)) \leq \eta_{\alpha} J_{\alpha}(w, T(w))\right\}$. Hence, since $\forall_{\alpha \in A}\left\{\eta_{\alpha} \in[0,1 / 2)\right\}$, we get

$$
\forall_{\alpha \in \mathcal{A}}\left\{J_{\alpha}(w, T(w))=0\right\}
$$

Similarly, by $(\mathcal{J} 1)$ and $(\mathrm{C} 2)$, we obtain $\forall_{\alpha \in \mathcal{A}} \exists_{\eta_{\alpha} \in[0,1 / 2)} \forall_{m \in \mathbb{N}}\left\{J_{\alpha}(T(w), w) \leq J_{\alpha}\left(T(w), T\left(v^{m}\right)\right)+J_{\alpha}\left(T\left(v^{m}\right), w\right) \leq \eta_{\alpha}\left[J_{\alpha}(T(w), w)+J_{\alpha}\left(v^{m}, v^{m+1}\right)\right]+J_{\alpha}\left(v^{m+1}, w\right)\right\}$. Consequently, by Step 1 and (D2), we have $\forall_{\alpha \in \mathcal{A}} \exists_{\eta_{\alpha} \in[0,1 / 2)}\left\{J_{\alpha}(T(w), w) \leq \eta_{\alpha} J_{\alpha}(T(w), w)+\lim _{m \rightarrow \infty}\left[\eta_{\alpha} J_{\alpha}\left(v^{m}, v^{m+1}\right)+J_{\alpha}\left(v^{m+1}, w\right)\right]=\eta_{\alpha} J_{\alpha}(T(w), w)\right\}$ and, since $\forall_{\alpha \in \mathcal{A}}\left\{\eta_{\alpha} \in[0,1 / 2)\right\}, \forall_{\alpha \in \mathcal{A}} \exists_{\eta_{\alpha} \in[0,1 / 2)}\left\{J_{\alpha}(T(w), w) \leq \eta_{\alpha} J_{\alpha}(T(w), w)\right\}$ implies

$$
\forall_{\alpha \in \mathcal{A}}\left\{J_{\alpha}(T(w), w)=0\right\}
$$

From (5.1), (5.2) and Remark 3.1, we conclude that $T(w)=w$.

Case 2. If (C3) holds, then by $(\mathcal{J} 1)$ and $(\mathrm{C} 3)$, we have that $\forall_{\alpha \in \mathcal{A}} \exists_{\eta_{\alpha} \in[0,1 / 2)} \forall_{m \in \mathbb{N}}\left\{J_{\alpha}(w, T(w)) \leq J_{\alpha}\left(w, T\left(v^{m}\right)\right)+J_{\alpha}\left(T\left(v^{m}\right), T(w)\right) \leq J_{\alpha}\left(w, v^{m+1}\right)+\eta_{\alpha}\left[J_{\alpha}\left(v^{m}, v^{m+1}\right)+J_{\alpha}(w, T(w))\right]\right\} . \quad$ Consequently, by (D2) and Step $\forall_{\alpha \in \mathcal{A}} \exists_{\eta_{\alpha} \in[0,1 / 2)}\left\{\lim _{m \rightarrow \infty} J_{\alpha}(w, T(w)) \leq \lim _{m \rightarrow \infty}\left[J_{\alpha}\left(w, v^{m+1}\right)+\eta_{\alpha} J_{\alpha}\left(v^{m}, v^{m+1}\right)+\eta_{\alpha} J_{\alpha}(w, T(w))\right]=\eta_{\alpha} J_{\alpha}(w, T(w))\right\}$. Therefore, $\forall_{\alpha \in \mathcal{A}} \exists_{\eta_{\alpha} \in[0,1 / 2)}\left\{J_{\alpha}(w, T(w)) \leq \eta_{\alpha} J_{\alpha}(w, T(w))\right\}$, so, since $\forall_{\alpha \in \mathcal{A}}\left\{\eta_{\alpha} \in[0,1 / 2)\right\}$, we get

$$
\forall_{\alpha \in \mathcal{A}}\left\{J_{\alpha}(w, T(w))=0\right\} .
$$

Similarly,

$\forall_{\alpha \in \mathcal{A}} \exists_{\eta_{\alpha} \in[0,1 / 2)} \forall_{m \in \mathbb{N}}\left\{J_{\alpha}(T(w), w) \leq J_{\alpha}\left(T(w), T\left(v^{m}\right)\right)+J_{\alpha}\left(T\left(v^{m}\right), w\right) \leq \eta_{\alpha}\left[J_{\alpha}(w, T(w))+J_{\alpha}\left(v^{m}, v^{m+1}\right)\right]+J_{\alpha}\left(v^{m+1}, w\right)\right\}$. Consequently, by (5.3), Step 1 and (D2), $\forall_{\alpha \in \mathcal{A}} \exists_{\eta_{\alpha} \in[0,1 / 2)}\left\{J_{\alpha}(T(w), w) \leq \eta_{\alpha} J_{\alpha}(w, T(w))+\lim _{m \rightarrow \infty}\left[\eta_{\alpha} J_{\alpha}\left(v^{m}, v^{m+1}\right)+J_{\alpha}\left(v^{m+1}, w\right)\right]=0\right\}$. Therefore,

$$
\forall_{\alpha \in \mathcal{A}}\left\{J_{\alpha}(T(w), w)=0\right\}
$$

From (5.3), (5.4) and Remark 3.1, we conclude that $T(w)=w$.

Case 3. If (C4) holds, then by $(\mathcal{J} 1)$ and $(\mathrm{C} 4)$, we have that $\forall_{\alpha \in \mathcal{A}} \exists_{\eta_{\alpha} \in[0,1 / 2)} \forall_{m \in \mathbb{N}}\left\{J_{\alpha}(T(w), w) \leq J_{\alpha}\left(T(w), T\left(v^{m}\right)\right)+J_{\alpha}\left(T\left(v^{m}\right), w\right) \leq \eta_{\alpha}\left[J_{\alpha}(w, T(w))+J_{\alpha}\left(v^{m+1}, v^{m}\right)\right]+J_{\alpha}\left(v^{m+1}, w\right)\right\}$. Consequently, by (D2) and Step 1,

$$
\forall_{\alpha \in \mathcal{A}} \exists_{\eta_{\alpha} \in[0,1 / 2)}\left\{J_{\alpha}(T(w), w) \leq \eta_{\alpha} J_{\alpha}(w, T(w)) \leq J_{\alpha}(w, T(w))\right\} .
$$

$$
\text { However, by }(\mathcal{J} 1) \text { and }(\mathrm{C} 4) \text {, we }
$$
$\forall_{\alpha \in \mathcal{A}} \exists_{\eta_{\alpha} \in[0,1 / 2)} \forall_{m \in \mathbb{N}}\left\{J_{\alpha}(w, T(w)) \leq J_{\alpha}\left(w, T\left(v^{m}\right)\right)+J_{\alpha}\left(T\left(v^{m}\right), T(w)\right) \leq J_{\alpha}\left(w, v^{m+1}\right)+\eta_{\alpha}\left[J_{\alpha}\left(v^{m}, v^{m+1}\right)+J_{\alpha}(T(w), w)\right]\right\}$. Consequently, by Step 1 and (D2), 


$$
\forall_{\alpha \in \mathcal{A}} \exists_{\eta_{\alpha} \in[0,1 / 2)}\left\{J_{\alpha}(w, T(w)) \leq \eta_{\alpha} J_{\alpha}(T(w), w) \leq J_{\alpha}(T(w), w)\right\}
$$

Clearly, (5.5) and (5.6) give

$$
\forall_{\alpha \in \mathcal{A}}\left\{J_{\alpha}(T(w), w)=J_{\alpha}(w, T(w))\right\}
$$

If, there exists $\alpha_{0} \in \mathcal{A}$, such that $J_{\alpha_{0}}(w, T(w))=J_{\alpha_{0}}(T(w), w)>0$, then using the above consideration, by $(\mathcal{J} 1)$ and $(\mathrm{C} 4)$, since $\eta_{\alpha_{0}} \in[0,1 / 2)$, we obtain $0<J_{\alpha_{0}}(w, T(w)) \leq \eta_{\alpha_{0}} J_{\alpha_{0}}(T(w), w)<J_{\alpha_{0}}(T(w), w)=J_{\alpha_{0}}(w, T(w))$, which is absurd. Consequently, we have that

$$
\forall_{\alpha \in \mathcal{A}}\left\{J_{\alpha}(T(w), w)=J_{\alpha}(w, T(w))=0\right\} .
$$

From (5.8) and Remark 3.1, we conclude that $T(w)=w$.

Clearly, by $(\mathcal{J} 1)$ and (D2), we have $\forall_{\alpha \in \mathcal{A}}\left\{J_{\alpha}(w, w) \leq \lim _{m \rightarrow \infty} J_{\alpha}\left(w, v^{m}\right)+\lim _{m \rightarrow \infty} J_{\alpha}\left(v^{m}, w\right)=0\right\}$.

STEP 4. If $v^{0}, w \in X$. satisfy (D2), then $\forall_{\alpha \in \mathcal{A}} \forall_{u^{0} \in X}\left\{\lim _{m \rightarrow \infty} J_{\alpha}\left(u^{m}, w\right)=0\right\}$.

Indeed, we consider three cases:

Case 1. If (C2) holds, then, by (J1), (C2), Proposition 3.4(b), Step 1 and (D2), we conclude

that

$\forall_{\alpha \in \mathcal{A}} \exists_{\eta_{\alpha} \in[0,1 / 2)} \forall_{u^{0} \in \mathcal{X}\{}\left[\lim _{m \rightarrow \infty} J_{\alpha}\left(u^{m}, w\right) \leq \lim _{m \rightarrow \infty}\left[J_{\alpha}\left(u^{m}, v^{m}\right)+J_{\alpha}\left(v^{m}, w\right)\right] \leq \eta_{\alpha}\left[\lim _{m \rightarrow \infty}\left(I_{\alpha}\left(u^{m}, u^{m-1}\right)+J_{\alpha}\left(v^{m-1}, v^{m}\right)\right)\right]+\lim _{m \rightarrow \infty} J_{\alpha}\left(v^{m}, w\right)=0\right\}, \quad$ SO $\forall_{\alpha \in \mathcal{A}} \forall_{u^{0} \in X}\left\{\lim _{m \rightarrow \infty} J_{\alpha}\left(u^{m}, w\right)=0\right\}$ holds.

Case 2. If (C3) holds, then, by (J1), (C3), Proposition 3.4(b), Step 1 and (D2), we conclude that

$\forall_{\alpha \in \mathcal{A}} \exists_{\eta_{\alpha} \in[0,1 / 2) \forall_{u^{0}} \in \chi}\left\{\left(\lim _{m \rightarrow \infty} J_{\alpha}\left(u^{m}, w\right) \leq \lim _{m \rightarrow \infty}\left[J_{\alpha}\left(u^{m}, v^{m}\right)+J_{\alpha}\left(v^{m}, w\right)\right] \leq \eta_{\alpha}\left[\lim _{m \rightarrow \infty}\left(J_{\alpha}\left(u^{m-1}, u^{m}\right)+J_{\alpha}\left(v^{m-1}, v^{m}\right)\right)\right]+\lim _{m \rightarrow \infty} J_{\alpha}\left(v^{m}, w\right)=0\right\}\right.$,

$\forall_{\alpha \in \mathcal{A}} \forall_{u^{0} \in X}\left\{\lim _{m \rightarrow \infty} J_{\alpha}\left(u^{m}, w\right)=0\right\}$ holds.

Case 3. If (C4) holds, then, by (J1), (C4), Proposition 3.4(b), Step 1 and (D2), we conclude

that

$\forall_{\alpha \in \mathcal{A}} \exists_{\eta_{\alpha} \in[0,1 / 2)} \forall_{u^{0} \in \mathcal{X}}\left[\lim _{m \rightarrow \infty} J_{\alpha}\left(u^{m}, w\right) \leq \lim _{m \rightarrow \infty}\left[J_{\alpha}\left(u^{m}, v^{m}\right)+J_{\alpha}\left(v^{m}, w\right)\right] \leq \eta_{\alpha}\left[\lim _{m \rightarrow \infty}\left(J_{\alpha}\left(u^{m-1}, u^{m}\right)+J_{\alpha}\left(v^{m}, v^{m-1}\right)\right)\right]+\lim _{m \rightarrow \infty} J_{\alpha}\left(v^{m}, w\right)=0\right\}$, so

$\forall_{\alpha \in \mathcal{A}} \forall_{u^{0} \in X}\left\{\lim _{m \rightarrow \infty} J_{\alpha}\left(u^{m}, w\right)=0\right\}$ holds.

STEP 5. If $v^{0}, w \in X$ satisfy (D2), then $\forall_{\alpha \in \mathcal{A}} \forall_{u^{0} \in X}\left\{\lim _{n \rightarrow \infty} d_{\alpha}\left(u^{n}, w\right)=0\right\}$.

Assume that at least one of the conditions (C2) - (C4) holds. Then, by Proposition 3.4(a), Step 4 and (J2), $\forall_{\alpha \in \mathcal{A}} \forall_{u^{0} \in X}\left\{\lim _{m \rightarrow \infty} d_{\alpha}\left(u^{m}, w\right)=0\right\}$.

STEP 6. If $v^{0}, w \in X$ satisfy (D2), then $\operatorname{Fix}(T)=\{w\}$.

Indeed, assume that at least one of the conditions (C2)-(C4) holds. Then, by Step 3 and Proposition 3.4(d), we have that $\operatorname{Fix}(T)=\{w\}$.

STEP 7. The assertions (a)-(c) are satisfied.

This is a consequence of Steps 6, 5 and 3.

\section{Proof of Theorem 2.3}

The proof will be broken into six steps.

STEP 1. If $v^{0} \in X$ and $w \in X$ satisfy (D1), then

$$
\lim _{m \rightarrow \infty} v^{m}=w
$$

This follows from Proposition 3.4(e).

STEP 2. If at least one of the conditions (C2)-(C4) holds and, additionally, the conditions (D1) and (D3) hold, then (D2) is satisfied. 
Assume that $v^{0} \in X$ and $w \in X$ satisfy (Dl). By (6.1) and (D3), we have that $w=T^{[\mathrm{q}]}$ (w) for some $q \in \mathbb{N}$. Next, by (C2) or (C3) or (C4) and by Proposition 3.4(d), Fix (T) = $\{w\}$ and $\forall_{\alpha \in \mathcal{A}}\left\{J_{\alpha}(w, w)=0\right\}$. This and $T(w)=w$, by (C2) or (C3) or (C4) and Proposition 3.4(b), imply: (i) $\lim _{m \rightarrow \infty} J_{\alpha}\left(w, v^{m}\right)=\lim _{m \rightarrow \infty} J_{\alpha}\left(T(w), T\left(v^{m-1}\right)\right) \leq \eta_{\alpha} \lim _{m \rightarrow \infty}\left[J_{\alpha}(T\right.$ $\left.(w), w)+J_{\alpha}\left(v^{m-1}, v^{m}\right)\right]=\eta_{\alpha} \lim _{m \rightarrow \infty}\left[J_{\alpha}(w, w)+J_{\alpha}\left(v^{m-1}, v^{m}\right)\right]=0$ if (C2) holds; (ii) $\lim _{m \rightarrow \infty} J_{\alpha}\left(w, v^{m}\right)=\lim _{m \rightarrow \infty} J_{\alpha}\left(T(w), T\left(v^{m-1}\right)\right) \leq \eta_{\alpha} \lim _{m \rightarrow \infty}\left[J_{\alpha}(w, T(w))+J_{\alpha}\left(v^{m-1}, v^{m}\right)\right]$ $=\eta_{\alpha} \lim _{m \rightarrow \infty}\left[J_{\alpha}(w, w)+J_{\alpha}\left(v^{m-1}, v^{m}\right)\right]=0$ if (C3) holds; (iii) $\lim _{m \rightarrow \infty} J_{\alpha}\left(w, v^{m}\right)=$ $\lim _{m \rightarrow \infty} J_{\alpha}\left(T(w), T\left(v^{m-1}\right)\right) \leq \eta_{\alpha} \lim _{m \rightarrow \infty}\left[J_{\alpha}(w, T(w))+J_{\alpha}\left(v^{m}, v^{m-1}\right)\right]=\eta_{\alpha} \lim _{m \rightarrow \infty}\left[J_{\alpha}(w\right.$, $\left.w)+J_{\alpha}\left(v^{m}, v^{m-1}\right)\right]=0$ if (C4) holds. This and (D1) imply (D2).

STEP 3. If at least one of the conditions (C2)-(C4) holds and, additionally, the conditions (D1) and (D4) hold, then (D2) is satisfied.

Let us observe that (D3), by assumption (Dl), includes (D4). Indeed, if $v^{0} \in X$ and $w$ $\in X$ satisfy (Dl) and if (D4) holds, then, by (6.1), $T^{[q]}$ is continuous at $w$ for some $q \in$ $\mathbb{N}$ and, since $v^{m q+k}=T^{[q]}\left(v^{(m-1) q+k}\right)$ for $k=1,2, \ldots, q$ and $m \in \mathbb{N}$ and, for each $k=1,2, \ldots$ $q$, the sequences $\left(v^{m+q+k}: m \in\{0\} \cup \mathbb{N}\right)$ and $\left(v^{m-q+k}: m \in\{0\} \cup \mathbb{N}\right)$, as subsequences of $\left(v^{m}: m \in\{0\} \cup \mathbb{N}\right)$, also converge to $w$, we obtain that $w=T^{[\mathrm{q}]}(w)$. By Step 2, (D2) holds.

STEP 4. If at least one of the conditions (C2)-(C4) holds and, additionally, the conditions (D1) and (D5) hold, then (D2) is satisfied.

Indeed, if $v^{0} \in X$ and $w \in X$ satisfy (Dl) and if (D5) holds, then $\lim _{m \rightarrow \infty} \nu^{m}=T^{[q]}(w)$ and, since $X$ is Hausdorff, by (6.1), we obtain $w=T^{[q]}(w)$, i.e. (D3) holds. By Step 2, (D2) holds.

STEP 5. If at least one of the conditions (C2)-(C4) holds and, additionally, the conditions (D1) and (D6) hold, then (D2) is satisfied.

Let $v^{0} \in X$ and $w \in X$ satisfy (Dl). Then, by (C2) or (C3) or (C4) and Proposition 3.4 (b), we have that $\forall_{\alpha \in \mathcal{A}}\left\{\lim _{n \rightarrow \infty} \sup _{m>n} J_{\alpha}\left(v^{n}, v^{m}\right)=0\right\}$ and, by (6.1) and (D6), it follows that $\exists_{q \in \mathbb{N}} \forall_{\alpha \in \mathcal{A}}\left\{\lim _{m \rightarrow \infty} J_{\alpha}\left(v^{m}, T^{[q]}(w)\right)=0\right\}$. Defining $x_{m}=v^{m}$ and $y_{m}=T^{[q]}(w)$ for $m \in \mathbb{N}$, we conclude that for sequences $\left(x_{m}: m \in \mathbb{N}\right)$ and $\left(y_{m}=m \in \mathbb{N}\right)$ in $X$ the conditions (2.1) and (2.2) hold. Hence, by $(\mathcal{J} 2)$, we get (2.3) which implies $\forall_{\alpha \in \mathcal{A}}\left\{\lim _{m \rightarrow \infty} d_{\alpha}\left(v^{m}, T^{[q]}(w)\right)=0\right\}$, i.e. $\lim _{m \rightarrow \infty} v^{\mathrm{m}}=T^{[q]}(w)$. Since $X$ is Hausdorff and (6.1) holds, this gives $w=T^{[q]}(w)$, i.e. (D3) holds. By Step 2, (D2) holds.

STEP 6. The assertions (a)-(c) are satisfied.

This is a consequence of Steps 1-5 and Theorem 2.2.

The proof of Theorem 2.3 is complete.

\section{Proof of Theorem 2.4}

The proof will be broken into five parts.

PART 1. Since $X$ is a Hausdorff sequentially complete uniform space and, by $(\mathrm{Cl})$ or (C2) or (C3) or (C4) and by Proposition 3.4(c), for each $u^{0} \in X$, the sequence $\left(u^{m}: m\right.$ $\in\{0\} \cup \mathbb{N}$ ) is a Cauchy sequence, thus there exists a unique $w \in X$ such that $\lim _{m \rightarrow \infty}$ $u^{m}=w$

PART 2. If $u^{0} \in X, \lim _{m \rightarrow \infty} u^{m}=w$ and (D3) holds, then we have that $w \in F i x\left(T^{[q]}\right)$ for some $q \in \mathbb{N}$. Next, by Proposition 3.4(d), we conclude that the other assertions hold. 
PART 3. If $u^{0} \in X, \lim _{m \rightarrow \infty} u^{m}=w$ and (D4) holds, i.e. $T^{[q]}$ is continuous at $w$ for some $q \in \mathbb{N}$, then $w=T^{[q]}(w)$. Indeed, we have that $u^{m q+k}=T^{[q]}\left(u^{(m-1) q+k}\right)$ for $k=$ $1,2, \ldots, q$ and $m \in \mathbb{N}$ and, for each $k=1,2, \ldots, q$, the sequences $\left(u^{m q+k}: m \in\{0\} \cup \mathbb{N}\right)$ and $\left(u^{(m-1) q+k}: m \in\{0\} \cup \mathbb{N}\right)$, as subsequences of $\left(u^{m}: m \in\{0\} \cup \mathbb{N}\right)$, also converge to $w$, which, since $T^{[q]}$ is continuous at $w$, gives $w=T^{[q]}(w)$. Hence, using Proposition 3.4(d), we conclude that the other assertions hold.

PART 4. If $u^{0} \in X, \lim _{m \rightarrow \infty} u^{m}=w$ and (D5) holds, then we obtain that $\lim _{m \rightarrow \infty} u^{m}$ $=T^{[q]}(w)$, which, with Part 1 , since $X$ is Hausdorff, gives $w=T^{[q]}(w)$, i.e. (D3) holds.

PART 5. If $u^{0} \in X, \lim _{m \rightarrow \infty} u^{m}=w$ and (D6) holds, then we obtain that $\exists_{q \in \mathbb{N}} \forall_{\alpha \in \mathcal{A}}\left\{\lim _{m \rightarrow \infty} J_{\alpha}\left(u^{m}, T^{[q]}(w)\right)=0\right\}$ and next, using Proposition 3.4(a) and (J2), we conclude that $\lim _{m \rightarrow \infty} u^{m}=T^{[q]}(w)$, which, with Part 1 , gives $w=T^{[q]}(w)$, i.e. (D3) holds.

The proof of Theorem 2.4 is complete.

\section{Proof of Theorem 2.5}

The proof will be broken into four steps.

STEP 1. Let at least one of the conditions (C1)-(C4) holds and let $u^{0} \in X$ be arbitrary and fixed. Then:

$$
\begin{aligned}
& \forall_{\alpha \in \mathcal{A}}\left\{\lim _{n \rightarrow \infty} \sup _{m>n} J_{\alpha}\left(u^{n}, u^{m}\right)=0\right\}, \\
& \exists_{w \in X} \forall_{\alpha \in \mathcal{A}}\left\{\lim _{m \rightarrow \infty} J_{\alpha}\left(u^{m}, w\right)=\lim _{m \rightarrow \infty} J_{\alpha}\left(w, u^{m}\right)=0\right\}, \\
& \forall_{\alpha \in \mathcal{A}}\left\{\lim _{m \rightarrow \infty} J_{\alpha}\left(u^{m}, u^{m+1}\right)=\lim _{m \rightarrow \infty} J_{\alpha}\left(u^{m+1}, u^{m}\right)=0\right\} .
\end{aligned}
$$

Indeed, (8.1) is a consequence of Proposition 3.4(a). Property (8.1) and Definition 2.2 imply (8.2). Proposition 3.3(d) implies (8.3).

STEP 2. Let (C1) hold and let $u^{0} \in X$ and $w \in X$ be such as in the Step 1. Then, Fix $(T)=\{w\}$ and $\forall_{\alpha \in \mathcal{A}}\left\{J_{\alpha}(w, w)=0\right\}$, i.e. the assertions (a) and (c) hold.

First, we show that

$$
\forall_{\alpha \in \mathcal{A}}\left\{J_{\alpha}(T(w), w)=0\right\}
$$

and

$$
\forall_{\alpha \in \mathcal{A}}\left\{J_{\alpha}(w, T(w))=0\right\} .
$$

Suppose that $\exists_{\alpha_{0} \in \mathcal{A}}\left\{J_{\alpha_{0}}(T(w), w)>0\right\}$. By $(\mathcal{J} 1)$ and $(\mathrm{Cl})$, we obtain that

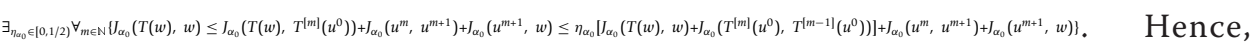
using (8.3) and (8.2), $\left.J_{\alpha_{0}}(T(w), w)=\lim _{m \rightarrow \infty} J_{\alpha_{0}}(T(w), w) \leq \eta_{\alpha_{0}} \lim _{m \rightarrow \infty} \mid \|_{\alpha_{0}}(T(w), w)++_{\alpha_{0}}\left(u^{m}, u^{m-1}\right)\right)+\lim _{m \rightarrow \infty} \mid\left[\alpha_{\alpha_{0}}\left(u^{m}, u^{m+1}\right)++_{\alpha_{0}}\left(u^{m+1}, w\right)\right]=\eta_{\alpha_{0}} J_{\alpha_{0}}(T(w), w) . \quad$ Thus, since $\eta_{\alpha_{0}} \in[0,1 / 2)$, we get $J_{\alpha_{0}}(T(w), w)=0$, which is impossible. Therefore, (8.4) holds.

Next, we see that, for arbitrary and fixed $\alpha \in \mathcal{A}$, by $(\mathcal{J} 1)$ and $(\mathrm{Cl})$, $\exists_{\eta_{e} \in[0,1 / 2)} \forall_{m \in \mathbb{N}}\left\{J_{\alpha}(w, T(w)) \leq J_{\alpha}\left(w, u^{m}\right)+J_{\alpha}\left(T^{|m|}\left(u^{0}\right), T(w)\right) \leq I_{\alpha}\left(w, u^{m}\right)+\eta_{\alpha}\left[I_{\alpha}\left(T^{[m]}\left(u^{0}\right), T^{[m-1]}\left(u^{0}\right)\right)+J_{\alpha}(T(w), w)\right]\right\}$. Hence, using (8.2)-(8.4), we get $J_{\alpha}(w, T(w))=\lim _{m \rightarrow \infty} J_{\alpha}\left(w, T(w, T(w)) \leq \lim _{m \rightarrow \infty} J_{\alpha}\left(w, u^{m}\right)+\right.$ $\eta_{\alpha} \lim _{m \rightarrow \infty}\left[U_{\alpha}\left(u^{m}, u^{m-1}\right)+J_{\alpha}(T(w), w)\right]=0$, i.e. (8.5) holds. 
Now, from (8.4), (8.5) and Remark 3.1, we obtain that $w=T(w)$, i.e. $w \in F i x(T)$. Hence, by Proposition 3.4(d) (for $q=1$ ), we have that $\operatorname{Fix}(T)=\{w\}$ and $\forall_{\alpha \in \mathcal{A}}\left\{J_{\alpha}(w, w)=0\right\}$, i.e. the assertions (a) and (c) hold.

STEP 3. Let at least one of the conditions (C2)-(C4) holds and let $u^{0} \in X$ and $w \in X$ be such as in the Step 1. Then, Fix $(T)=\{w\}$ and $\forall_{\alpha \in \mathcal{A}}\left\{J_{\alpha}(w, w)=0\right\}$, i.e. the assertions (a) and (c) hold.

Indeed, the assertions (a) and (c) are consequences of (8.2), (8.3) and also consequences of similar argumentation as in Case 1 (if (C2) holds) or Case 2 (if (C3) holds) or Case 3 (if (C4) holds) of Step 3 of the proof of Theorem 2.2.

STEP 4. Let at least one of the conditions (C1)-(C4) holds. Then, $\forall_{u^{0} \in X}\left\{\lim _{m \rightarrow \infty} u^{m}=w\right\}$.

This is a consequence of (8.1), (8.2) and (J2).

\section{Proof of Theorem 2.6}

Let $q, w, v^{0}$ and $\left(v^{m_{k}}: k \in\{0\} \cup \mathbb{N}\right)$ be such as in (D7) and (D8).

Therefore,

$$
\lim _{k \rightarrow \infty} v^{m_{k}}=w
$$

and, by $(\mathrm{Cl})$ or $(\mathrm{C} 2)$ or $(\mathrm{C} 3)$ or $(\mathrm{C} 4)$ and by Proposition 3.4(c), we get, in particular,

$$
\forall \forall_{\alpha \in \mathcal{A}}\left\{\lim _{n \rightarrow \infty} \sup _{m>n} d_{\alpha}\left(v^{n}, v^{m}\right)=0\right\} .
$$

First, we show that

$$
\lim _{m \rightarrow \infty} v^{m}=w
$$

Indeed,

we

have

that

$\forall_{\alpha \in \mathcal{A}} \forall_{n \in \mathbb{N}} \exists_{p(n) \in \mathbb{N}, m_{p(n)} \geq n} \forall_{k>p(n)}\left\{d_{\alpha}\left(v^{n}, w\right) \leq d_{\alpha}\left(v^{n}, v^{m_{k}}\right)+d_{\alpha}\left(v^{m_{k}}, w\right) \leq \sup _{m>n} d_{\alpha}\left(v^{n}, v^{m}\right)+d_{\alpha}\left(v^{m_{k}}, w\right)\right\}$ which, by implies

that

$\forall_{\alpha \in \mathcal{A}} \forall_{n \in \mathbb{N}}\left\{d_{\alpha}\left(v^{n}, w\right) \leq \sup _{m>n} d_{\alpha}\left(v^{n}, v^{m}\right)+\lim _{k \rightarrow \infty} d_{\alpha}\left(v^{m_{k}}, w\right)=\sup _{m>n} d_{\alpha}\left(v^{n}, v^{m}\right)\right\}$. Hence, by (9.2), it follows that $\forall_{\alpha \in \mathcal{A}}\left\{\lim _{n \rightarrow \infty} d_{\alpha}\left(v^{n}, w\right) \leq \lim _{n \rightarrow \infty} \sup _{m>n} d_{\alpha}\left(v^{n}, v^{m}\right)=0\right\}$. Therefore, (9.3) holds.

Next, we see that

$$
w=T^{[q]}(w) .
$$

Indeed, we have $v^{m q+k}=T^{[q]}\left(v^{(m-1) q+k}\right)$ for $k=1,2, \ldots, q$ and $m \in \mathbb{N}$. Hence, by (D7) and (9.3), we get (9.4).

Now, we see that (9.4), Proposition 3.4(d) and (9.3) imply the assertions.

\section{Proof of Theorem 2.7}

If $\mathcal{J}=\mathcal{D}$, then $(\mathrm{Cl})=(\mathrm{Cl})=(\mathrm{C} 2)=(\mathrm{C} 3)=(\mathrm{C} 4)=(\mathrm{C} 5)$ and, by Proposition 3.4(c), we obtain that $\forall_{\alpha \in \mathcal{A}} \forall_{u^{0} \in X}\left\{\lim _{n \rightarrow \infty} \sup _{m>n} d_{\alpha}\left(u^{n}, u^{m}\right)=0\right\}$. Hence, since $X$ is a Hausdorff sequentially complete uniform space, it follows that if $u^{0} \in X$ is arbitrary and fixed, then the sequence $\left(u^{m}: m \in\{0\} \cup \mathbb{N}\right)$ is a Cauchy sequence and there exists a unique $w \in X$ such that $\forall_{\alpha \in \mathcal{A}}\left\{\lim _{m \rightarrow \infty} d_{\alpha}\left(u^{m}, w\right)=\lim _{m \rightarrow \infty} d_{\alpha}\left(w, u^{m}\right)=0\right\}$, i.e., for $\mathcal{J}=\mathcal{D}$, 
the conditions (D1) and (D2) hold. Using now Theorems 2.1 or 2.2, we get the assertions (a)-(c).

\section{Proof of Theorem 2.8}

Let $q, w, v^{0}$ and $\left(v^{m_{k}}: k \in\{0\} \cup \mathbb{N}\right)$ be such as in (D7) and (D8).

Using Theorem 2.6 for $\mathcal{J}=\mathcal{D}$, we obtain that $\operatorname{Fix}(T)=\{w\}$ and

$$
\lim _{m \rightarrow \infty} v^{m}=w
$$

Next, by Proposition 3.4(b), we get

$$
\forall_{\alpha \in \mathcal{A}} \forall_{u^{0} \in X}\left\{\lim _{n \rightarrow \infty} d_{\alpha}\left(u^{n+1}, u^{n}\right)=\lim _{n \rightarrow \infty} d_{\alpha}\left(u^{n}, u^{n+1}\right)=0\right\} .
$$

Moreover, by Proposition 3.4(c), we get, in particular, that

$$
\forall_{\alpha \in \mathcal{A}}\left\{\lim _{n \rightarrow \infty} \sup _{m>n} d_{\alpha}\left(v^{n}, v^{m}\right)=0 .\right.
$$

Finally, by (C5) and (11.

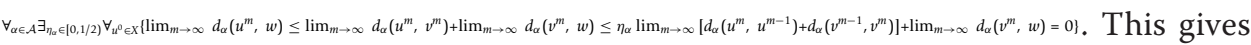
$\forall_{u^{0} \in X}\left\{\lim _{m \rightarrow \infty} u^{m}=w\right\}$.

\section{Examples and comparisons}

First, in the following, we record some conclusions on metric spaces.

Definition 12.1 Let $(X, d)$ be a metric space. The map $J: X^{2} \rightarrow[0, \infty)$ is said to be a J-generalized pseudodistance on $X$ if the following two conditions hold:

(J') $\left.\forall_{x, y}, z \in \mathrm{X}(x, z) \leq J(x, y)+J(y, z)\right\}$; and

(J2') For any sequences $\left(x_{m}: m \in \mathbb{N}\right)$ and $\left(y_{m}: m \in \mathbb{N}\right.$ in $X$ such that

$$
\lim _{n \rightarrow \infty} \sup _{m>n} J\left(x_{n}, x_{m}\right)=0
$$

and

$$
\lim _{m \rightarrow \infty} J\left(x_{m}, y_{m}\right)=0,
$$

the following holds

$$
\lim _{m \rightarrow \infty} d\left(x_{m}, y_{m}\right)=0 \text {. }
$$

Theorems 2.1, 2.2 and 2.4 imply:

Theorem 12.1 Let $(X, d)$ be a metric space. Assume that the map $T: X \rightarrow X$ and the $J$-generalized pseudodistance $J: X^{2} \rightarrow[0, \infty)$ on $X$ satisfy

$\left(C 1^{\prime}\right) \exists_{\eta \in[0,1 / 2)} \forall_{x, y \in X}\{J(T(x), T(y)) \leq \eta[J(T(x), x)+J(T(y), y)]\}$

and, additionally,

(D1') $\exists_{v^{0}, w \in X}\left\{\lim _{m \rightarrow \infty} J\left(v^{m}, w\right)=0\right\}$.

Then: (a) $T$ has a unique fixed point $w$ in $X ;(\mathbf{b}) \forall_{u^{0} \in X}\left\{\lim _{m \rightarrow \infty} u^{m}=w\right\}$; and (c) $J(w$, $w)=0$.

Theorem 12.2 Let $(X, d)$ be a metric space. Assume that the map $T: X \rightarrow X$ and the $J$-generalized pseudodistance $J: X^{2} \rightarrow[0, \infty)$ on $X$ satisfy at least one of the following three conditions:

$\left(C 2^{\prime}\right) \exists_{\eta \in[0,1 / 2)} \forall_{x, y \in X}\{J(T(x), T(y)) \leq \eta[J(T(x), x)+J(y, T(y))]\}$, 
(C3) $\exists_{\eta \in[0,1 / 2)} \forall_{x, y \in X}\{J(T(x), T(y)) \leq \eta[J(x, T(x))+J(y, T(y))]\}$,

(C4') $\exists_{\eta \in[0,1 / 2)} \forall_{x, y \in X}\{J(T(x), T(y)) \leq \eta[J(x, T(x))+J(T(y), y)]\}$,

and, additionally,

(D2') $\exists_{v^{0}, w \in X}\left\{\lim _{m \rightarrow \infty} J\left(v^{m}, w\right)=\lim _{m \rightarrow \infty} J\left(w, v^{m}\right)=0\right\}$.

Then: (a) Thas a unique fixed point $w$ in $X$; (b) $\forall_{u^{0} \in X}\left\{\lim _{m \rightarrow \infty} u^{m}=w\right\}$; and (c) $J(w$, $w)=0$.

Theorem 12.3 Let $(X, d)$ be a complete metric space and assume that the map $T: X$ $\rightarrow X$ and the J-generalized pseudodistance $J: X^{2} \rightarrow[0, \infty)$ on $X$ satisfy at least one of the conditions (C2')-(C4') and, additionally, condition (D1') and at least one of the following conditions (D3')-(D6'):

(D3') $\forall_{v^{0}, w \in X}\left\{\lim _{m \rightarrow \infty} v^{m}=w \Rightarrow \exists_{q \in \mathbb{N}}\left\{T^{[q]}(w)=w\right\}\right\}$,

(D4') $\forall_{v^{0}, w \in X}\left\{\lim _{m \rightarrow \infty} v^{m}=w \Rightarrow \exists_{q \in \mathbb{N}}\left\{T^{[q]}\right.\right.$ is continuous at a point $\left.\left.w\right\}\right\}$,

(D5') $\forall_{v^{0}, w \in X}\left\{\lim _{m \rightarrow \infty} v^{m}=w \Rightarrow \exists_{q \in \mathbb{N}}\left\{\lim _{m \rightarrow \infty} T^{[q]}\left(v^{m}\right)=T^{[q]}(w)\right\}\right\}$,

(D6') $\forall_{v^{0}, w \in X}\left\{\lim _{m \rightarrow \infty} v^{m}=w \Rightarrow \exists_{q \in \mathbb{N}}\left\{\lim _{m \rightarrow \infty} J\left(T^{[q]}\left(v^{m}\right), T^{[q]}(w)\right)=0\right\}\right\}$.

Then: (a) Thas a unique fixed point $w$ in $X ;(\mathbf{b}) \forall_{u^{0} \in X}\left\{\lim _{m \rightarrow \infty} u^{m}=w\right\}$; and (c) $J(w$, $w)=0$.

Now, we present some examples illustrating the concepts introduced so far.

First, we present an example of symmetric $J$-generalized pseudodistance.

Example 12.1. Let $X$ be a metric space with metric $d$. Let the set $E \subset X$, containing at least two different points, be arbitrary and fixed and let $c>0$ satisfy $\delta(E)<c$ where $\delta(E)=\sup \{d(x, y): x, y \in E\}$. Let $J: X^{2} \rightarrow[0, \infty)$ be defined by the formula

$$
J(x, y)=\left\{\begin{array}{ll}
d(x, y) & \text { if } E \cap\{x, y\}=\{x, y\}, \\
c & \text { if } E \cap\{x, y\} \neq\{x, y\},
\end{array}, y \in X .\right.
$$

We show that $J$ is a generalized pseudodistance on $X$.

Indeed, it is worth noticing that the condition $\left(J 1^{\prime}\right)$ does not hold only if there exist some $x_{0}, y_{0}, z_{0} \in X$ such that $J\left(x_{0}, z_{0}\right)>J\left(x_{0}, y_{0}\right)+J\left(y_{0}, z_{0}\right)$. This inequality is equivalent to $c>d\left(x_{0}, y_{0}\right)+d\left(y_{0}, z_{0}\right)$ where $J\left(x_{0}, z_{0}\right)=c, J\left(x_{0}, y_{0}\right)=d\left(x_{0}, y_{0}\right)$ and $J\left(y_{0}, z_{0}\right)=d\left(y_{0}\right.$, $\left.z_{0}\right)$. However, by (12.4): $J\left(x_{0}, z_{0}\right)=c$, gives that there exists $v \in\left\{x_{0}, z_{0}\right\}$ such that $v \notin E$; $J\left(x_{0}, y_{0}\right)=d\left(x_{0}, y_{0}\right)$ gives $\left\{x_{0}, y_{0}\right\} \subset E$; $J\left(y_{0}, z_{0}\right)=d\left(y_{0}, z_{0}\right)$ gives $\left\{y_{0}, z_{0}\right\} \subset E$. This is impossible. Therefore, $\forall_{x, y}, z \in X\{(x, y) \leq J(x, z)+J(z, y)\}$, i.e. the condition $\left(J 1^{\prime}\right)$ holds.

For proving that $\left(J 2^{\prime}\right)$ holds we assume that the sequences $\left(x_{m}: m \in \mathbb{N}\right)$ and $\left(y_{m}: m\right.$ $\in \mathbb{N}$ ) in $X$ satisfy (12.1) and (12.2). Then, in particular, (12.2) yields

$$
\forall_{0<\varepsilon<c} \exists_{m_{0}=m_{0}(\varepsilon) \in \mathbb{N}} \forall_{m \geq m_{0}}\left\{J\left(x_{m}, y_{m}\right)<\varepsilon\right\} .
$$

By (12.5) and (12.4), since $\varepsilon<c$, we conclude that

$$
\forall_{m \geq m_{0}}\left\{E \cap\left\{x_{m}, y_{m}\right\}=\left\{x_{m}, y_{m}\right\}\right\} .
$$

From (12.6), (12.4) and (12.5), we get

$$
\forall_{0<\varepsilon<c} \exists_{m_{0} \varepsilon \mathbb{N}} \forall_{m \geq m_{0}}\left\{d\left(x_{m}, y_{m}\right)=J\left(x_{m}, y_{m}\right)<\varepsilon\right\} .
$$

Therefore, the sequences $\left(x_{m}: m \in \mathbb{N}\right)$ and $\left(y_{m}: m \in \mathbb{N}\right)$ satisfy (12.3).

Consequently, the property $\left(J 2^{\prime}\right)$ holds.

Now, we define non-symmetric $J$-generalized pseudodistance. 
Example 12.2. Let $X=\mathbb{N}$ be a metric space with metric $d(x, y)=|x-y|$, $x, y \in X$. Let $a, b, c, d \in(0, \infty)$ be arbitrary and fixed and satisfying

$$
a<b<c<d .
$$

Let $J: X^{2} \rightarrow[0, \infty)$ be defined by the formula

$$
J(m, n)= \begin{cases}0 & \text { if } m=n, \\ b & \text { if } m \text { - even, } n \text { - even, } m \neq n, \\ c & \text { if } m \text { - odd, } n \text { - odd, } m \neq n, \quad x, y \in X . \\ d & \text { if } m \text { - even, } n \text { - odd, } \\ a & \text { if } m \text { - odd, } n \text { - even, }\end{cases}
$$

We show that $J$ is a generalized pseudodistance on $X$.

Indeed, first we show that the condition $\left(J 1^{\prime}\right)$ holds. Let $m, n, s \in \mathbb{N}$ be arbitrary and fixed. We consider the following cases:

Case 1. If $m$-even and $n$-odd, then: (i) if $s$-even, we have $J(m, n)=d, J(m, s)=b, J(s$, $n)=d$, and, consequently, $J(m, n) \leq J(m, s)+J(s, n)$; (ii) if $s$-odd, we have $J(m, n)=d$, $J$ $(m, s)=d, J(s, n)=c$, and, consequently, $J(m, n) \leq J(m, s)+J(s, n)$.

Case 2. If $m$-even and $n$-even, then: (i) if $s$-even, we have $J(m, n)=b, J(m, s)=b, J(s$, $n)=b$, and, consequently, $J(m, n) \leq J(m, s)+J(s, n)$; (ii) if $s$-odd, we have $J(m, n)=b, J$ $(m, s)=d, J(s, n)=a$, and, consequently, by (12.7), we get $J(m, n) \leq J(m, s)+J(s, n)$.

Case 3. If $m$-odd and $n$-even, then: (i) if $s$-even, we have $J(m, n)=a, J(m, s)=a, J(s$, $n)=b$, and, consequently, $J(m, n) \leq J(m, s)+J(s, n)$; (ii) if $s$-odd, we have $J(m, n)=a, J$ $(m, s)=c, J(s, n)=a$, and, consequently, $J(m, n) \leq J(m, s)+J(s, n)$.

Case 4. If $m$-odd and $n$-odd, then: (i) if $s$-even, we have $J(m, n)=c, J(m, s)=a, J(s$, $n)=d$, and, consequently, by (12.7), we get $J(m, n) \leq J(m, s)+J(s, n)$; (ii) if $s$-odd, we have $J(m, n)=c, J(m, s)=c, J(s, n)=c$, and, consequently, $J(m, n) \leq J(m, s)+J(s, n)$.

Thus, $J$ satisfies the condition $\left(J 1^{\prime}\right)$.

For proving that $\left(J 2^{\prime}\right)$ holds we assume that the sequences $\left(x_{m}: m \in \mathbb{N}\right)$ and $\left(y_{m}: m\right.$ $\in \mathrm{N})$ in $X$ satisfy (12.1) and (12.2). Then, in particular, (12.2) yields

$$
\forall_{0<\varepsilon<a} \exists_{m_{0}=m_{0}(\varepsilon) \in \mathbb{N}} \forall_{m \geq m_{0}}\left\{J\left(x_{m}, y_{m}\right)<\varepsilon\right\} .
$$

By (12.9) and (12.8), since $\varepsilon<a$, we conclude that

$$
\forall_{m \geq m_{0}}\left\{x_{m}=y_{m}\right\} .
$$

From (12.10), (12.8) and (12.9), we get

$$
\forall_{0<\varepsilon<a} \exists_{m_{0} \in \mathbb{N}} \forall_{m \geq m_{0}}\left\{d\left(x_{m}, y_{m}\right)=0<\varepsilon\right\} .
$$

Therefore, the sequences $\left(x_{m}: m \in \mathbb{N}\right)$ and $\left(y_{m}: m \in \mathbb{N}\right)$ satisfy (12.3). Consequently, the property $\left(J 2^{\prime}\right)$ holds.

Next, we present an example of noncomplete metric space $X, J$-generalized pseudodistances on $X$ and map $T: X \rightarrow X$ such that Theorems 12.1 and 12.2 hold.

Example 12.3. Let $X=[0,1)$ be a noncomplete metric space with a metric $d: X^{2} \rightarrow$ $[0, \infty), d(x, y)=|x-y|, x, y \in X$. Let $T: X \rightarrow X$ be a map given by the formula

$$
T(x)= \begin{cases}0 & \text { if } x=1 / 4 \\ 1 / 2 & \text { if } x \in X \backslash\{1 / 4\}\end{cases}
$$


Let $E=[0,1 / 4) \cup[1 / 2,3 / 4)$ and let

$$
J(x, y)= \begin{cases}d(x, y) & \text { if }\{x, y\} \cap E=\{x, y\} \\ 2 & \text { if }\{x, y\} \cap E \neq\{x, y\} .\end{cases}
$$

The map $J$ is a generalized pseudodistance on $X$ (see Example 12.1). Clearly, $\forall_{v^{0} \in X} \forall_{m \geq 2}\left\{v^{m}=1 / 2=T^{[m]}\left(v^{0}\right) \in E\right\}, w=1 / 2 \in E$ and $\forall_{m} \geq 2\left\{J\left(v^{m}, w\right)=J\left(w, v^{m}\right)=d\right.$ $\left.\left(v^{m}, w\right)=0\right\}$. Hence, we conclude that the conditions (D1') and (D2') hold.

We observe that $T$ satisfies conditions: (C1')-(C4'). Indeed, for each $x \in X$, by (12.11), we have that $T(x) \in E$, so $J(T(x), T(y))=d(T(x), T(y))=d(1 / 2,1 / 2)=0$ if $\{x, y\} \cap\{1 / 4\}$ $=\varnothing, J(T(x), T(y))=d(T(x), T(y))=1 / 2$ if $\{x \neq y \wedge\{x, y\} \cap\{1 / 4\} \neq \varnothing\}$ and $J(T(x), T(y))=$ $d(T(x), T(y))=0$ if $x=y=1 / 4$. Consequently, there exists $\lambda=3 / 8 \in[0,1 / 2)$ such that for each $x, y \in X$, since $1 / 4 \notin E$, using (12.12) we have

$$
\begin{aligned}
& J(T(x), T(y)) \\
= & \begin{cases}0 \leq \frac{3}{8}[J(T(x), x)+J(T(y), y)] & \text { if }\{x, y\} \cap\left\{\frac{1}{4}\right\}=\emptyset, \\
\frac{1}{2}<\frac{3}{4} \leq \frac{3}{8}[J(T(x), x)+J(T(y), y)] & \text { if } x \neq y,\{x, y\} \cap\left\{\frac{1}{4}\right\} \neq \emptyset, \\
0 \leq \frac{3}{8}[J(T(x), x)+J(T(y), y)] & \text { if } x=y=\frac{1}{4} .\end{cases}
\end{aligned}
$$

Here, we use the fact that if $x \neq y$ and $\{x, y\} \cap\{1 / 4\} \neq \varnothing$, then $J(T(x), x)=2$ or $J(y, T$ $(y))=J(T(y), y)=2$. Therefore, $\left(C 1^{\prime}\right)$ holds. By formula (12.12), $J$ is symmetric. Hence, $\left(\mathrm{C1}^{\prime}\right)=\left(\mathrm{C}^{\prime}\right)=\left(\mathrm{C} 3^{\prime}\right)=\left(\mathrm{C} 4^{\prime}\right)$.

Assertions (a)-(c) of Theorems 12.1 and 12.2 hold: we have that $\operatorname{Fix}(T)=\{1 / 2\}$, $\forall_{u^{0} \in X}\left\{\lim _{m \rightarrow \infty} u^{m}=1 / 2\right\}$ and $J(1 / 2,1 / 2)=d(1 / 2,1 / 2)=0$.

It is worth noticing that the conditions (D1') in Theorem 12.1 and (D2') in Theorem 12.2 cannot be omitted.

Example 12.4. Let $X=[0,1)$ be a noncomplete metric space with a metric $d: X^{2} \rightarrow$ $[0, \infty), d(x, y)=|x-y|, x, y \in X$. Let $T: X \rightarrow X$ be a map given by the formula $T(x)=$ $x / 4+3 / 4, x \in X$.

From Remark 2.1(b), it follows that the map $J=d$ is a generalized pseudodistance on $X$.

We observe that the conditions (C1')-(C4') hold.

Indeed, let $\eta=1 / 3$ and let $x, y \in X$ be arbitrary and fixed.

If $x<y$, then, since $T$ is strictly increasing, we have $T(x)<T(y)$ and, consequently,

$$
d(T(x), T(y))=d(x / 4+3 / 4, y / 4+3 / 4)=y / 4-x / 4 .
$$

Moreover, $\forall_{z \in X}\{d(T(z), z)=z / 4+3 / 4-z=-(3 / 4) z+3 / 4\}$. Hence,

$$
\begin{aligned}
\frac{1}{3}[d(T(x), x)+d(T(y), y)] & =\frac{1}{3}\left[-\frac{3 x}{4}+\frac{3}{4}-\frac{3 y}{4}+\frac{3}{4}\right] \\
& =\frac{1}{3}\left[\frac{3}{2}-\frac{3 x}{4}-\frac{3 y}{4}\right]=\frac{1}{2}-\frac{x}{4}-\frac{y}{4} .
\end{aligned}
$$

From this, we conclude that $d(T(x), T(y)) \leq \eta[d(T(x), x)+d(T(y), y)]$. Indeed, otherwise $d(T(x), T(y))>\eta[d(T(x), x)+d(T(y), y)]$ and then, by (12.13) and (12.14), we must have that $y / 4-x / 4>1 / 2-x / 4-y / 4$. However, this gives $y>1$, which is absurd.

If $x>y$, then, by analogous considerations, we obtain a similar conclusion.

Therefore, $\exists_{\eta}=1 / 3 \forall_{x, y \in X}\{d(T(x), T(y)) \leq \eta[d(T(x), x)+d(T(y), y)]\}$, i.e. the conditions (C1')-(C4') hold. 
Clearly, for each $u^{0} \in X$, the sequence $\left.\left(u^{m}: m \in\{0\} \cup \mathbb{N}\right)\right\}$, where $u^{m}=T^{[m]}\left(u^{0}\right), m$ $\in \mathbb{N}$, is not convergent in $X$, so, the conditions (D1') and (D2') do not hold. We see that $\operatorname{Fix}(T)=\varnothing$.

We illustrate Theorem 12.3.

Example 12.5. Let $(X, d)$ be a metric space, where $X=[0,1]$ and $d(x, y)=|x-y|, x$, $y \in X$. Let $T: X \rightarrow X$ be a map given by the formula

$$
T(x)= \begin{cases}0 & \text { if } x=1 / 4 \\ 1 / 2 & \text { if } x \in X \backslash\{1 / 4\}\end{cases}
$$

Clearly, the map $T^{2}, T^{2}(x)=1 / 2, x \in X$, is continuous on $X$, so the condition (D4') holds.

Let $E=[0,1 / 4) \cup[1 / 2,3 / 4)$ and let

$$
J(x, y)= \begin{cases}d(x, y) & \text { if }\{x, y\} \cap E=\{x, y\} \\ 2 & \text { if }\{x, y\} \cap E \neq\{x, y\} .\end{cases}
$$

By Example 12.1, $J$ is a $J$-generalized pseudodistance. Using analogous considerations as in Example 12.3, we prove that $T$ and $J$ satisfy conditions ( $\left.\mathrm{C1}^{\prime}\right)-\left(\mathrm{C} 4^{\prime}\right)$.

The above implies that Theorem 12.3 holds. Assertions (a)-(c) are as follows: $w=1 / 2, \operatorname{Fix}(T)=\{w\}, \forall_{u^{0} \in X}\left\{\lim _{m \rightarrow \infty} u^{m}=w\right\}$ and $J(w, w)=d(w, w)=0$.

We notice that the existence of $J$-generalized pseudodistance such that $\mathcal{J} \neq d$ is essential.

Example 12.6. Let $X$ and $T$ be such as in Examples 12.3 or 12.5. We observe that $T$ is not Kannan contraction, i.e. $T$ does not satisfy (K). Indeed, suppose that

$$
\exists_{\eta \in[0,1 / 2)} \forall_{x, y \in X}\{d(T(x), T(y)) \leq \eta[d(T(x), x)+d(T(y), y)]\} .
$$

Then, in particular, for $x_{0}=1 / 4$ and $y_{0}=3 / 4$ from $X$, by (12.15), we obtain $1 / 2=d$ $(0,1 / 2)=d\left(T\left(x_{0}\right), T\left(y_{0}\right)\right) \leq \eta\left[d\left(T\left(x_{0}\right), x_{0}\right)+d\left(T\left(y_{0}\right), y_{0}\right)\right]=\eta[d(0,1 / 4)+d(1 / 2,3 / 4)]$ $=\eta(1 / 4+1 / 4)<1 / 2$, which is absurd.

In Examples 12.7 and 12.8, we compare conditions (C1')-(C4') and (K).

Example 12.7. Let $X=\{2,3,5\}$ be a metric space with a metric $d: X^{2} \rightarrow[0, \infty), d(x$, $y)=|x-y|, x, y \in X$. Let $T: X \rightarrow X$ be a map given by the formula

$$
T(m)=\left\{\begin{array}{l}
5 \text { if } m=2, \\
3 \text { if } m \in X \backslash\{2\} .
\end{array}\right.
$$

Define

$$
J(m, n)=\left\{\begin{array}{l}
0 \quad \text { if } m=n, \\
1 / 4 \text { if } m \text { - even, } n \text { - even, } m \neq n, \\
1 / 3 \text { if } m \text { - odd, } n \text { - odd, } m \neq n, \quad x, y \in X . \\
1 \quad \text { if } m \text { - even, } n \text { - odd, } \\
1 / 7 \text { if } m \text { - odd, } n \text { - even, }
\end{array}\right.
$$

The map $J$ is a generalized pseudodistance on $X$ (see Example 12.2). Next, we see that $\forall_{v^{0} \in X} \forall_{m \geq 2}\left\{v^{m}=3=T^{[m]}\left(v^{0}\right)\right\}, w=3 \in X$ and $\forall_{m} \geq 2\left\{J\left(v^{m}, w\right)=J\left(w, v^{m}\right)=d\left(v^{m}\right.\right.$, $w)=0\}$. Hence, we conclude that the conditions (D1') and (D2') hold.

We also observe that $T$ satisfies conditions (C3'). Indeed, let $\eta=1 / 3 \in[0,1 / 2)$, let $x$, $y \in X$ be arbitrary and fixed and consider the following five cases: 
Case 1. If $x=2$ and $y=3$, then, using (12.16) and (12.17), we obtain: $T(x)=5 ; T(y)$ $=3 ; J(T(x), T(y))=J(5,3)=1 / 3 \leq 1 / 3=(1 / 3)(1+0)=(1 / 3)[J(2,5)+J(3,3)]=(1 / 3) U$ $(x, T(x))+J(y, T(y))]$.

Case 2. If $x=3$ and $y=2$, then, using (12.16) and (12.17), we obtain: $T(x)=3 ; T(y)$ $=5 ; J(T(x), T(y))=J(3,5)=1 / 3 \leq 1 / 3=(1 / 3)(0+1)=(1 / 3)[J(3,3)+J(2,5)]=(1 / 3)[J$ $(x, T(x))+J(y, T(y))]$.

Case 3. If $x=2$ and $y=5$, then, using (12.16) and (12.17), we obtain: $T(x)=5 ; T(y)$ $=3 ; J(T(x), T(y))=J(5,3)=1 / 3 \leq 4 / 9=(1 / 3)(1+1 / 3)=(1 / 3)[J(2,5)+J(5,3)]=(1 / 3)$ $[(x, T(x))+J(y, T(y))]$.

Case 4. If $x=5$ and $y=2$, then, using (12.16) and (12.17), we obtain: $T(x)=3 ; T(y)$ $=5 ; J(T(x), T(y))=J(3,5)=1 / 3 \leq 4 / 9=(1 / 3)(1 / 3+1)=(1 / 3)[J(5,3)+J(2,5)]=(1 / 3)$ $[(x, T(x))+J(y, T(y))]$.

Case 5. If $x=3$ and $y=5$ or $x=5$ and $y=3$, then, using (12.16) and (12.17), in both situations, we obtain: $T(x)=3 ; T(y)=3 ; J(T(x), T(y))=J(3,3)=0 \leq(1 / 3)[J(x, T(x))+J$ $(y, T(y))]$.

Therefore, the condition (C3') holds.

Assertions (a)-(c) of Theorems 12.1 and 12.2 hold: we have that $\operatorname{Fix}(T)=\{3\}$, $\forall_{u^{0} \in X}\left\{\lim _{m \rightarrow \infty} u^{m}=3\right\}$ and $J(3,3)=0$.

Example 12.8. Let $X, J, T$ be as in Example 12.7.

First, we show that $T$ and $J$ do not satisfy the condition (C1'). Indeed, suppose that $\exists_{\eta \in[0,1 / 2)} \forall_{x, y \in X}\{(T(x), T(y)) \leq \eta[J(T(x), x)+J(T(y), y)]\}$. Hence, in particular, for $x$ $=2$ and $y=3$, we get $1 / 3=J(5,3)=J(T(x), T(y)) \leq \eta[J(T(x), x)+J(T(y), y)]=\eta[J(5,2)$ $+J(3,3)]=\eta[1 / 7+0]<1 / 14$, absurd.

Now, we show that $T$ and $J$ does not satisfy the condition (C2'). Indeed, suppose that $\exists_{\eta \in[0,1 / 2)} \forall_{x, y \in X}\{(T(x), T(y)) \leq \eta[J(T(x), x)+J(y, T(y))]\}$. Hence, in particular, for $x$ $=2$ and $y=3$, we get $1 / 3=J(5,3)=J(T(x), T(y)) \leq \eta[J(T(x), x)+J(y, T(y))]=\eta[J(5,2)$ $+J(3,3)]=\eta[1 / 7+0]<1 / 14$, absurd.

Next, we show that $T$ and $J$ do not satisfy the condition (C4'). Indeed, suppose that $\exists_{\eta \in[0,1 / 2)} \forall_{x, y \in X}\{J(T(x), T(y)) \leq \eta[J(x, T(x))+J(T(y), y)]\}$. Hence, in particular, for $x$ $=3$ and $y=2$, we get $1 / 3=J(3,5)=J(T(x), T(y)) \leq \eta[J(x, T(x))+J(T(y), y)]=\eta[J(3,3)$ $+J(5,2)]=\eta[0+1 / 7]<1 / 14$, absurd.

Finally, we observe that $T$ do not satisfy the condition (K). Indeed, suppose that $\exists_{\eta \in}$ $[0,1 / 2) \forall_{x, y \in X}\{d(T(x), T(y)) \leq \eta[d(T(x), x)+d(T(y), y)]\}$. Hence, in particular, for $x=2$ and $y=3$, we get $2=d(5,3)=d(T(x), T(y)) \leq \eta[J(T(x), x)+J(T(y), y)]=\eta[d(5,2)+d$ $(3,3)]=\eta[3+0]<3 / 2$, absurd.

\section{Conclusion}

Now, we present some conclusions:

(a) Let $X$ be a Hausdorff sequentially complete uniform space. If $\mathcal{J}=\mathcal{D}$ and $T$ satisfies (C5), then (C1)-(C5) are identical and, by Proposition 3.4(c), for each $u^{0} \in X$, a sequence $\left(u^{m}: m \in\{0\} \cup \mathbb{N}\right)$ is Cauchy and thus convergent. By Definition 2.2, this gives that $T$ is $\mathcal{D}$-admissible.

Hence, in particular, it follows that in complete metric spaces $(X, d)$, each Kannan contraction is $d$-admissible.

(b) Theorem 2.2 includes Theorem 2.3.

(c) Theorem 2.7 is a version of Theorem 1.2 for uniform spaces. 
(d) If $X$ is a Hausdorff sequentially complete uniform space, then, for $\mathcal{J}=\mathcal{D}$, Theorems 2.1 and 2.2 include Theorem 2.7.

(e) In metric spaces, for $\mathcal{J}=\mathcal{D}=\{d\}$, we conclude that: (i) Theorem 2.6 includes Theorem 1.3; (ii) Theorem 2.8 generalizes Theorem 1.3 (in Theorem 2.8, we have, additionally, the assertion (b)).

(f) Four kinds of Kannan-type contractions (C1')-(C4') where $J$ are $\tau$-distances are introduced and studied by Suzuki [[14], Contractions: (a) and (b), p. 199; (c) and (d), p. 200]. Our results are different from those given in [14].

(g) $\tau$-distances [10] and $\tau$-functions [22] are generalized pseudodistances but the converse does not holds; see [23-26].

(h) Conditions (C1)-(C4) are different; see e.g. Examples 12.2, 12.7 and 12.8.

\section{Authors' contributions}

The authors have equitably contributed in obtaining the new results presented in this article. All authors read and approved the final manuscript.

\section{Competing interests}

The authors declare that they have no competing interests.

Received: 8 June 2011 Accepted: 30 November 2011 Published: 30 November 2011

\section{References}

1. Banach, S: Sur les opérations dans les ensembles abstraits et leurs applications aux équations intégrales. Fund Math. 3, 133-181 (1922)

2. Caccioppoli, R: U teorema generale sull'esistenza di elementi uniti in una transformazione funzionale. Rend Accad dei Lincei. 11, 794-799 (1930)

3. Kannan, R: Some results on fixed points. Bull Calcutta Math Soc. 60, $71-76$ (1968)

4. Kannan, R: Some results on fixed points-II. Amer Math Mon. 76, 405-408 (1969). doi:10.2307/2316437

5. Connell, EH: Properties of fixed point spaces. Proc Amer Math Soc. 10, 974-979 (1959). doi:10.1090/S0002-9939-19590110093-3

6. Subrahmanyam, PV: Remarks on some fixed point theorems related to Banach's contraction principle. J Math Phys Sci 8, 445-458 (1974). Erratum 9, 195 (1975)

7. Suzuki, T: Contractive mappings are Kannan mappings, and Kannan mappings are contractive mappings in some sense. Comment Math. 45, 43-56 (2005)

8. Kikkawa, M, Suzuki, T: Some similarity between contractions and Kannan mappings. Fixed Point Theory Appl 2008, 1-8 (2008). Article ID 649749

9. Kada, O, Suzuki, T, Takahashi, W: Nonconvex minimization theorems and fixed point theorems in complete metric spaces. Math Japonica. 44, 381-391 (1996)

10. Suzuki, T: Generalized distance and existence theorems in complete metric spaces. J Math Anal Appl. 253, 440-458 (2001). doi:10.1006/jmaa.2000.7151

11. Nakanishi, M, Suzuki, T: An observation on Kannan mappings. Cent Eur J Math. 8, 170-178 (2010). doi:10.2478/s11533009-0065-9

12. Shioji, N, Suzuki, T, Takahashi, W: Contractive mappings, Kannan mappings and metric completeness. Proc Amer Math Soc. 126, 3117-3124 (1998). doi:10.1090/S0002-9939-98-04605-X

13. Suzuki, T: Several fixed point theorems in complete metric spaces. Yokohama Math J. 44, 61-72 (1997)

14. Suzuki, T: Several fixed point theorems concerning T-distance. Fixed Point Theory Appl. 2004, 195-209 (2004)

15. Suzuki, T: Subrahmanyam's fixed point theorem. Nonlinear Anal. 71, 1678-1683 (2009). doi:10.1016/j.na.2009.01.004

16. Suzuki, T: Convergence of the sequence of successive approximations to a fixed points. Fixed Point Theory Appl 2010, 1-14 (2010). Article ID 716971

17. Enjouji, Y, Nakanishi, M, Suzuki, T: A generalization of Kannan's fixed point theorem. Fixed Point Theory Appl 2009, 1-10 (2009). Article ID 192872

18. Leader, S, Hoyle, SL: Contractive fixed points. Fund Math. 87, 93-108 (1975)

19. Park, S: Characterizations of metric completeness. Colloq Math. 49, $21-26$ (1984)

20. Reich, S: Kannan's fixed point theorem. Boll Un Mat Ital. 4, 1-11 (1971)

21. Reich, S: Some remarks concerning contraction mappings. Canad Math Bull. 14, 121-124 (1971). doi:10.4153/CMB-1971024-9

22. Lin, L-J, Du, W-S: Ekeland's variational principle, minimax theorems and existence of nonconvex equilibria in complete metric spaces. J Math Anal Appl. 323, 360-370 (2006). doi:10.1016/j.jmaa.2005.10.005

23. Włodarczyk, K, Plebaniak, R: Maximality principle and general results of Ekeland and Caristi types without lower semicontinuity assumptions in cone uniform spaces with generalized pseudodistances. Fixed Point Theory Appl 2010 1-35 (2010). Article ID 175453

24. Włodarczyk, K, Plebaniak, R: Periodic point, endpoint, and convergence theorems for dissipative set-valued dynamic systems with generalized pseudodistances in cone uniform and uniform spaces. Fixed Point Theory Appl 2010, 1-32 (2010). Article ID 864536 
25. Włodarczyk, K, Plebaniak, R: Quasigauge spaces with generalized quasi-pseudodistances and periodic points of dissipative set-valued dynamic systems. Fixed Point Theory Appl 2011, 1-22 (2011). Article ID 712706

26. Włodarczyk, K, Plebaniak, R: Contractivity of Leader type and fixed points in uniform spaces with generalized pseudodistances. J Math Anal Appl. 387, 533-541 (2012). doi:10.1016/j.jmaa.2011.09.006

doi:10.1186/1687-1812-2011-90

Cite this article as: Włodarczyk and Plebaniak: Kannan-type contractions and fixed points in uniform spaces. Fixed Point Theory and Applications 2011 2011:90.

Submit your manuscript to a SpringerOpen ${ }^{\circ}$ journal and benefit from:

- Convenient online submission

- Rigorous peer review

- Immediate publication on acceptance

- Open access: articles freely available online

- High visibility within the field

- Retaining the copyright to your article

Submit your next manuscript at $\boldsymbol{\nabla}$ springeropen.com 